\title{
LIMIT THEOREMS FOR STATIONARY TESSELLATIONS WITH RANDOM INNER CELL STRUCTURES
}

\author{
LOTHAR HEINRICH, ${ }^{*}$ University of Augsburg \\ HENDRIK SCHMIDT ${ }^{* *}$ AND \\ VOLKER SCHMIDT, ${ }^{* * * * *}$ University of Ulm
}

\begin{abstract}
We consider stationary and ergodic tessellations $X=\left\{\Xi_{n}\right\}_{n \geq 1}$ in $\mathbb{R}^{d}$, where $X$ is observed in a bounded and convex sampling window $W_{\varrho} \subset \mathbb{R}^{d}$. It is assumed that the cells $\Xi_{n}$ of $X$ possess random inner structures, examples of which include point patterns, fibre systems, and tessellations. These inner cell structures are generated, both independently of each other and independently of the tessellation $X$, by generic stationary random sets that are related to a stationary random vector measure $J_{0}$ acting on $\mathbb{R}^{d}$. In particular, we study the asymptotic behaviour of a multivariate random functional, which is determined both by $X$ and by the individual cell structures contained in $W_{\varrho}$, as $W_{\varrho} \uparrow \mathbb{R}^{d}$. It turns out that this functional provides an unbiased estimator for the intensity vector associated with $J_{0}$. Furthermore, under natural restrictions, strong laws of large numbers and a multivariate central limit theorem of the normalized functional are proven. Finally, we discuss in detail some numerical examples and applications, for which the inner structures of the cells of $X$ are induced by iterated Poisson-type tessellations.
\end{abstract}

Keywords: Stationary random tessellation; inner random cell structure; cumulative random functional; ergodic theorem; central limit theorem; Poisson nesting; model choice; telecommunication network

2000 Mathematics Subject Classification: Primary 60D05

Secondary 60F05; 90B 15

\section{Introduction}

Let $X=\left\{\Xi_{n}\right\}_{n \geq 1}$ be a stationary and ergodic tessellation in $\mathbb{R}^{d}$. For each $n \geq 1$, consider a certain ( $d$-dimensional) random vector $\alpha\left(\Xi_{n}\right)$, which is called an associated point of the cell $\Xi_{n}$ of $X$. It is well known that the tessellation $X$ can be regarded as a stationary and ergodic marked point process in $\mathbb{R}^{d}$, written as a sum $\sum_{n \geq 1} \delta_{\left[\alpha\left(\Xi_{n}\right), \Xi_{n}^{0}\right]}$ of Dirac measures $\delta_{\left[\alpha\left(\Xi_{n}\right), \Xi_{n}^{0}\right]}$, where the shifted cells $\Xi_{n}^{0}=\Xi_{n}-\alpha\left(\Xi_{n}\right)$ contain the origin $o \in \mathbb{R}^{d}$; see, e.g. Section 6.1 of [21]. Furthermore, for each $n \geq 1$, consider a vector $J_{n}=\left(J_{n}^{(1)}, \ldots, J_{n}^{(m)}\right)^{\top}$ of $m \geq 1$ stationary random measures in $\mathbb{R}^{d}$. Assume that the sequence $\left(J_{n}\right)_{n \geq 1}$ is independent of $X$ and that it consists of independent, identically distributed copies of some generic random vector measure $J_{0}=\left(J_{0}^{(1)}, \ldots, J_{0}^{(m)}\right)^{\top}$.

For each $n \geq 1$, the random measures $J_{n}^{(1)}, \ldots, J_{n}^{(m)}$ describe the inner structure of the $n$th cell $\Xi_{n}$ of the tessellation $X$. In particular, in the planar case $d=2$, examples of such

Received 24 February 2004; revision received 22 September 2004.

* Postal address: Institute of Mathematics, University of Augsburg, D-86135 Augsburg, Germany.

** Postal address: Department of Stochastics, University of Ulm, D-89069 Ulm, Germany.

*** Email address: schmidt@mathematik.uni-ulm.de 


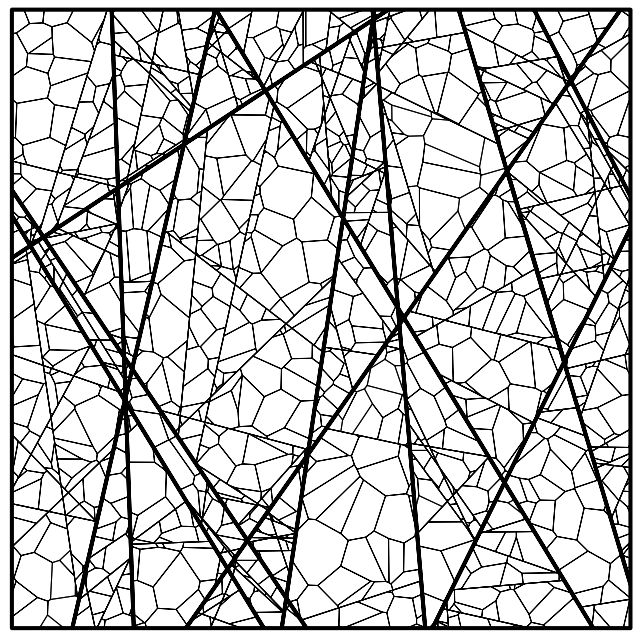

(a) A PLT/PVT-nesting

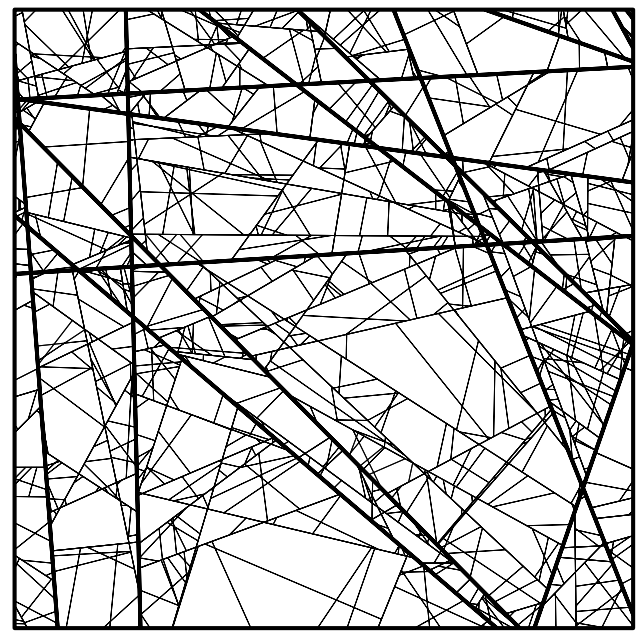

(b) A PLT/PLT-nesting

Figure 1: The inner structure of the cells of $X$, induced by iterated tessellations.

random measures are the number of vertices and the number, or the total length, of the edges, which are generated by some (component) tessellation $X_{n}$ within the cell $\Xi_{n}$ of the (initial) tessellation $X$. Figure 1(a) shows the case where $X_{n}$ is a so-called PLT/PVT-nesting, which means that Poisson line tessellations (PLTs) iterated by Poisson-Voronoi tessellations (PVTs) are inscribed into the cells of $X$. Similarly, in Figure 1(b), PLT/PLT-nestings inscribed into the cells of $X$ are shown. However, the random measures $J_{n}^{(1)}, \ldots, J_{n}^{(m)}$ need not necessarily be induced by tessellations. Another type of example is shown in Figure 2, where the inner structure of $\Xi_{n}$ is determined by point processes.

Suppose that only a single realization of the tessellation $X$, as well as of the vector measures $J_{1}, J_{2}, \ldots$, is available. This realization is restricted to some (presumably large) sampling window $W_{\varrho}$, and the support of $J_{n}$ is observable only in $\Xi_{n} \cap W_{\varrho}, n \geq 1$. The region $W_{\varrho}$ is assumed to have the form $W_{\varrho}=\varrho W$ with scaling factor $\varrho>0$ (which has no upper bound) and with a convex body $W \subset \mathbb{R}^{d}$ containing the closed ball $b(o, r)$ centred at the origin and of fixed radius $r>0$. The main subject investigated in the present paper is the vector of cumulative functionals $Z_{\varrho}=\left(Z_{\varrho}^{(1)}, \ldots, Z_{\varrho}^{(m)}\right)^{\top}$, where the components $Z_{\varrho}^{(i)}$ of $Z_{\varrho}$ are given by

$$
Z_{\varrho}^{(i)}=\sum_{n \geq 1} J_{n}^{(i)}\left(\Xi_{n} \cap W_{\varrho}\right)
$$

for each $i=1, \ldots, m$. As a first step, we determine the expectation vector $\mathrm{E} Z_{\varrho}$ and the covariance matrix $\operatorname{cov}\left(Z_{\varrho}\right)$ of $Z_{\varrho}$, as well as the asymptotic covariance matrix $K=\lim _{\varrho \rightarrow \infty} \operatorname{cov}\left(\widetilde{Z}_{\varrho}\right)$ of the vector of normalized functionals

$$
\widetilde{Z}_{\varrho}=\left(\frac{Z_{\varrho}^{(1)}-\lambda^{(1)}\left|W_{\varrho}\right|}{\sqrt{\left|W_{\varrho}\right|}}, \ldots, \frac{Z_{\varrho}^{(m)}-\lambda^{(m)}\left|W_{\varrho}\right|}{\sqrt{\left|W_{\varrho}\right|}}\right)^{\top} .
$$

Here, $|B|$ denotes the $d$-dimensional Lebesgue measure of any bounded Borel set $B \subset \mathbb{R}^{d}$ and $\lambda^{(i)}=\mathrm{E} J_{0}^{(i)}\left([0,1)^{d}\right)$ is the intensity of the stationary random measure $J_{0}^{(i)}$; see Theorem 3.1, below. 


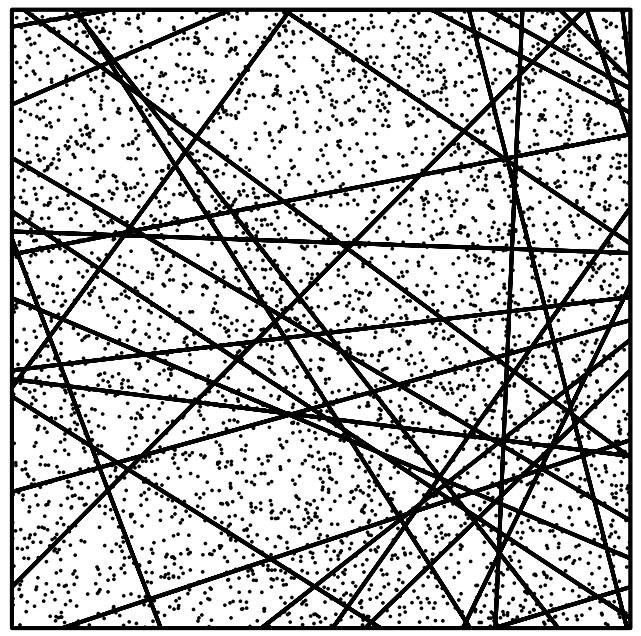

(a) Poisson point process

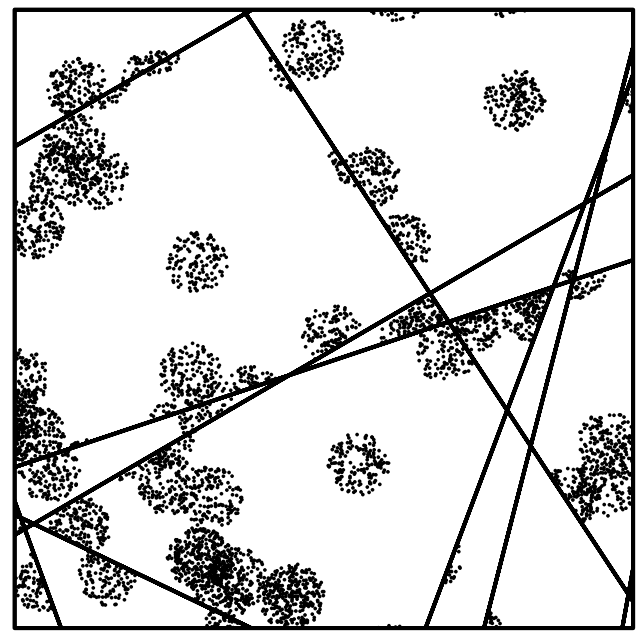

(b) Cluster point process

FIGURE 2: The inner structure of the cells of $X$, induced by point processes.

In the next step, under some mild integrability conditions, Theorem 4.2 yields the following strong law of large numbers:

$$
\frac{1}{\left|W_{\varrho}\right|} \sum_{n \geq 1} J_{n}^{(i)}\left(\Xi_{n} \cap W_{\varrho}\right) \underset{\varrho \rightarrow \infty}{\stackrel{\text { a.s. }}{\longrightarrow}} \lambda^{(i)} \quad \text { for } i=1, \ldots, m
$$

This shows that the vector $Z_{\varrho} /\left|W_{\varrho}\right|$ is a strongly consistent (and unbiased) estimator for the intensity vector $\left(\lambda^{(1)}, \ldots, \lambda^{(m)}\right)^{\top}$ of the stationary vector measure $J_{0}$. (Note that 'a.s.' stands for 'almost surely' or 'almost sure', depending on the context.) The proof of Theorem 4.2 relies on the ergodicity of the tessellation $X=\left\{\Xi_{n}\right\}_{n \geq 1}$ and the conditional independence of the random vectors $J_{1}\left(\Xi_{1} \cap W_{\varrho}\right), J_{2}\left(\Xi_{2} \cap W_{\varrho}\right), \ldots$ given the tessellation $X$. For this purpose, we need some estimates which show that the contribution of those cells of $X$ hitting the boundary $\partial W_{\varrho}$ is asymptotically negligible as $\varrho \rightarrow \infty$; see Lemma 4.1 , below.

In the third step, we derive the following multivariate central limit theorem:

$$
\widetilde{Z}_{\varrho} \underset{\varrho \rightarrow \infty}{\stackrel{\mathrm{D}}{\longrightarrow}} \mathrm{N}(o, K), \quad \text { that is, } \quad \lim _{\varrho \rightarrow \infty} \sup _{x \in \mathbb{R}^{m}}\left|\mathrm{P}\left(\widetilde{Z}_{\varrho} \leq x\right)-\Phi_{K}(x)\right|=0
$$

where $\stackrel{\text { D }}{\rightarrow}$ ' means convergence in distribution and $\Phi_{K}$ denotes the distribution function of the ( $m$-dimensional) Gaussian vector $\mathrm{N}(o, K)$ with zero-mean components and covariance matrix $K$; see Theorem 5.1, below.

Our results can be applied to stochastic modelling and statistical analysis of complex network structures. In [8], the so-called stochastic subscriber line model (SSLM) was described. This is an example of a stochastic-geometric model of telecommunication networks. Figure 3 shows a realization of the SSLM, in which the urban infrastructure along which the cable trench system is built is represented by a random tessellation. Within each cell, subscribers are located according to some point process, and line segments represent dead-end streets. Along the streets, 1-level 


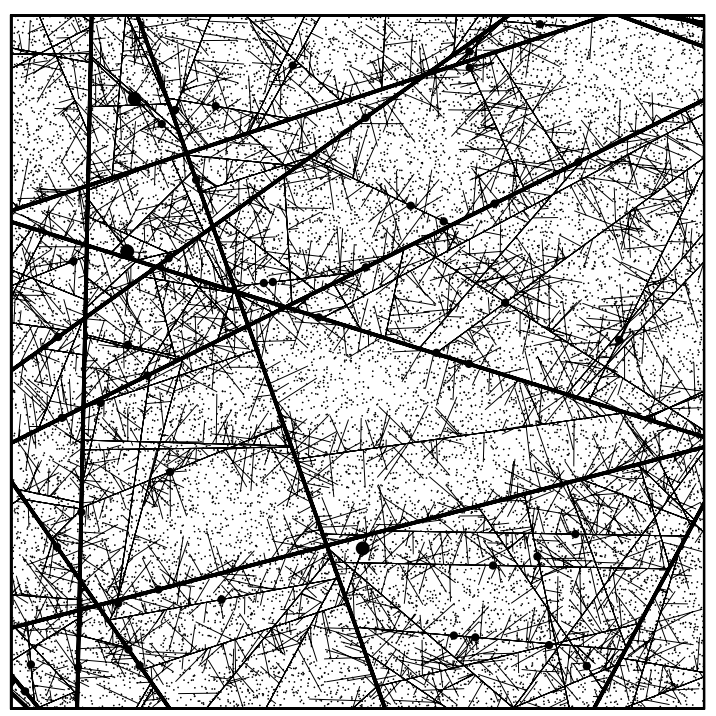

FIGURE 3: A realization of the stochastic subscriber line model.

and 2-level network stations are displayed (the small and the large dots, respectively). Each subscriber is connected along the road system to its closest (in the Euclidean sense) 2-level station via 1-level stations.

In the context of the SSLM, our results provide a theoretical basis for the statistical analysis of the morphological structure of spatial telecommunications data, and help fit appropriate tessellation models.

Notice that there exist a number of papers investigating problems closely related to the topics of our work. For example, in [10] a central limit theorem for a class of random measures associated with germ-grain models was derived, while, in [1] and [18] central limit theorems for Poisson-Voronoi and Poisson line tessellations in $\mathbb{R}^{2}$ were respectively investigated. In [9], normal approximations were given for some mean-value estimates of absolutely regular tessellations. Asymptotic properties of estimators for the volume fraction and other specific intrinsic volumes of stationary random sets were examined in, e.g. [3], [4], [14], and [20]. Simulation studies on the typical cell of stationary tessellations can be found, e.g. in [13].

The present paper is organized as follows. In Section 2, a short introduction to basic, general notions and notation of stochastic geometry is given. Section 3 is devoted to firstand second-order moments for functionals of stationary random measures associated with the cells of random tessellations. The strong law of large numbers and the multivariate central limit theorem mentioned above are derived in Sections 4 and 5, respectively. Some numerical examples are discussed in Section 6, where functionals are considered that describe several intracellular structures of the cells of tessellations in $\mathbb{R}^{2}$. Finally, in Section 7 , an outlook on directions for further research is given.

\section{Basic notions and notation}

In this section, the basic notation used in the present paper is introduced and a brief account of some relevant notions of stochastic geometry is given. For a detailed discussion of the subject, the reader is referred to the literature, for example [21] and [22]. 
The abbreviations int $B, \partial B$, and $B^{\mathrm{c}}$ are used to denote the interior, the boundary, and the complement of a set $B \subset \mathbb{R}^{d}$, respectively. For arbitrary sets $B, B^{\prime} \in \mathbb{R}^{d}$, we will consider the operations of translation: $B+x=\{y+x: y \in B\}$ for $x \in \mathbb{R}^{d}$; reflection: $\check{B}=-B=\{-x: x \in B\}$; scaling: $B_{\varrho}=\{\varrho x: x \in B\}$ for any constant $\varrho \in[0, \infty)$; and Minkowski-addition: $B \oplus B^{\prime}=\left\{x+x^{\prime}: x \in B, x^{\prime} \in B^{\prime}\right\}$. Furthermore, let $b(x, r)=$ $\left\{y \in \mathbb{R}^{d}:\|x-y\| \leq r\right\}$ denote the ball of radius $r \geq 0$ centred at $x \in \mathbb{R}^{d}$, where $\|x-y\|$ is the length of the vector $x-y$.

By $\mathcal{F}, \mathcal{K}$, and $\mathcal{C}$, the families of all closed sets, compact sets, and convex bodies (compact and convex sets) in $\mathbb{R}^{d}$ are denoted, respectively. For any $C \in \mathcal{C}$, the volume $|C \oplus b(o, r)|$ of the so-called parallel set $C \oplus b(o, r)$ is given by Steiner's formula

$$
|C \oplus b(o, r)|=\sum_{k=0}^{d}\left(\begin{array}{l}
d \\
k
\end{array}\right) M_{k}(C) r^{k}, \quad r \geq 0,
$$

where $M_{k}(C), 0 \leq k \leq d$, denotes the $k$ th Minkowski-functional of the convex body $C$. Note that, in particular, $M_{0}(C)=|C|$ and $M_{d}(C)=1-\delta_{\varnothing}(C)$, where $\delta_{\varnothing}$ denotes the Dirac measure on the empty set. Clearly, for each $\varrho>0$ and for any convex body $C \in \mathcal{C}$, the volume $\left|C_{\varrho}\right|$ equals $\varrho^{d}|C|$, and the diameter $D\left(C_{\varrho}\right)$ of the scaled set $C_{\varrho}$ is given by $\varrho D(C)$, where $D(C)=\sup \{\|x-y\|: x, y \in C\}$. Furthermore, for any $C \in \mathcal{C}$, the following isodiametric inequality holds (see, e.g. [11]):

$$
M_{k}(C) \leq \omega_{d}\left(\frac{D(C)}{2}\right)^{d-k}, \quad 0 \leq k \leq d,
$$

where $\omega_{d}$ denotes the volume of the unit ball $b(o, 1)$. Recall that a random closed set $\Xi$ in $\mathbb{R}^{d}$ is a measurable mapping $\Xi: \Omega \rightarrow \mathscr{F}$ from some probability space $(\Omega, \mathcal{A}, \mathrm{P})$ into the measurable space $(\mathcal{F}, \mathcal{B}(\mathcal{F}))$, where $\mathscr{B}(\mathcal{F})$ denotes the smallest $\sigma$-algebra of subsets of $\mathcal{F}$ that contains all sets $\{F \in \mathscr{F}: F \cap K \neq \varnothing\}$ for any $K \in \mathcal{K}$. In particular, the random closed set $\Xi$ is called a random compact set or a random convex body if $\mathrm{P}(\Xi \in \mathcal{K})=1$ or $\mathrm{P}(\Xi \in \mathcal{C})=1$, respectively.

A tessellation in $\mathbb{R}^{d}$ is a countable family $\tau=\left\{C_{n}\right\}_{n \geq 1}$ of convex bodies $C_{n} \in \mathcal{C}$ such that int $C_{n} \neq \varnothing$ for all $n$, int $C_{n} \cap$ int $C_{m}=\varnothing$ for all $n \neq m, \bigcup_{n \geq 1} C_{n}=\mathbb{R}^{d}$, and $\sum_{n \geq 1} \mathbf{1}_{\left\{C_{n} \cap K \neq \varnothing\right\}}$ $<\infty$ for any $K \in \mathcal{K}$ (where $\mathbf{1}_{\{\cdot\}}$ is an indicator function). Notice that the sets $C_{n}$, called the cells of $\tau$, are polytopes in $\mathbb{R}^{d}$. The family of all tessellations in $\mathbb{R}^{d}$ is denoted by $\mathcal{T}$. A random tessellation $X=\left\{\Xi_{n}\right\}_{n \geq 1}$ in $\mathbb{R}^{d}$ is a sequence of random convex bodies $\Xi_{n}$ such that $\mathrm{P}\left(\left\{\Xi_{n}\right\}_{n \geq 1} \in \mathcal{T}\right)=1$. Notice that a random tessellation $X$ can also be considered to be a marked point process $\sum_{n \geq 1} \delta_{\left[\alpha\left(\Xi_{n}\right), \Xi_{n}^{0}\right]}$, where $\alpha: \mathcal{C}^{\prime} \rightarrow \mathbb{R}^{d}$, with $\mathcal{C}^{\prime}=\mathcal{C} \backslash\{\varnothing\}$, is a measurable mapping such that $\alpha(C) \in C$ and $\alpha(C+x)=\alpha(C)+x$ for any $C \in \mathcal{C}^{\prime}$ and $x \in \mathbb{R}^{d}$, and where $\Xi_{n}^{0}=\Xi_{n}-\alpha\left(\Xi_{n}\right)$ is the centred cell corresponding to $\Xi_{n}$, and contains the origin. The point $\alpha(C) \in \mathbb{R}^{d}$ is called the associated point of $C$ and can be chosen, for example, to be the lexicographically smallest point of $C$; see, e.g. [16] and [17].

Suppose that the marked point process $\sum_{n \geq 1} \delta_{\left[\alpha\left(\Xi_{n}\right), \Xi_{n}^{0}\right]}$ is stationary with positive and finite intensity $\lambda=\operatorname{E} \operatorname{card}\left\{n: \alpha\left(\Xi_{n}\right) \in[0,1)^{d}\right\}$. By $\mathcal{P}^{0}$ we denote the set of all convex polytopes with their associated points at the origin. Then, the Palm mark distribution $P^{0}$ of $X$ is given by

$$
P^{0}(B)=\lambda^{-1} \mathrm{E} \operatorname{card}\left\{n: \alpha\left(\Xi_{n}\right) \in[0,1)^{d}, \Xi_{n}^{0} \in B\right\}, \quad B \in \mathcal{B}(\mathcal{F}) \cap \mathcal{P}^{0} .
$$


Notice that a random polytope $\Xi^{*}: \Omega \rightarrow \mathcal{P}^{0}$, whose distribution coincides with $P^{0}$, is called the typical cell of $X$. Furthermore, it holds that

$$
\lambda^{-1}=\int_{\mathcal{P}^{0}}|C| P^{0}(\mathrm{~d} C),
$$

i.e. the mean volume $\mathrm{E}\left|\Xi^{*}\right|=\int_{\mathcal{P}^{0}}|C| P^{0}(\mathrm{~d} C)$ of the typical cell $\Xi^{*}$ is equal to $\lambda^{-1}$.

A (deterministic) iterated tessellation $\tau=\left\{C_{n v} \cap C_{n}\right.$ : int $C_{n v} \cap$ int $\left.C_{n} \neq \varnothing\right\}$ in $\mathbb{R}^{d}$ consists of an initial tessellation $\tau=\left\{C_{n}\right\}_{n \geq 1}$ in $\mathbb{R}^{d}$ and a sequence $\left(\tau_{n}\right)_{n \geq 1}$ of component tessellations $\tau_{n}=\left\{C_{n v}\right\}_{\nu \geq 1}$. Hence, in order to define the notion of a random iterated tessellation, we can proceed as follows (see [12]). Let $\Xi$ be a random convex body in $\mathbb{R}^{d}$, where int $\Xi \neq \varnothing$, and let $X=\left\{\Xi_{n}\right\}_{n \geq 1}$ be a random tessellation in $\mathbb{R}^{d}$. Then, the mapping $Y(\cdot \mid \Xi): \Omega \rightarrow$ $N\left(\mathcal{F}^{\prime}\right)$, defined by $Y(B \mid \Xi)=\sum_{n \geq 1} \delta_{\Xi_{n} \cap \Xi}(B) \mathbf{1}_{\left\{\text {int } \Xi_{n} \cap \text { int } \Xi \neq \varnothing\right\}}$ for $B \in \mathscr{B}\left(\mathcal{F}^{\prime}\right)$, where $\mathcal{F}^{\prime}=\mathscr{F} \backslash\{\varnothing\}$, is a point process in $\mathcal{C}^{\prime}$. The space of all nonnegative and integer-valued measures on $\mathscr{B}\left(\mathcal{F}^{\prime}\right)$ is denoted by $N\left(\mathcal{F}^{\prime}\right)$, where each $\eta \in N\left(\mathcal{F}^{\prime}\right)$ can be represented by a finite or countable sum of Dirac measures $\delta_{F}$ of sets $F \in \mathcal{F}^{\prime}$, i.e. $\eta(B)=\sum_{n \geq 1} \eta\left(\left\{F_{n}\right\}\right) \delta_{F_{n}}(B)$ for any $B \in \mathcal{B}\left(\mathcal{F}^{\prime}\right)$, and $\eta(\{F \in \mathcal{F}: F \cap K \neq \varnothing\})<\infty$ for any $K \in \mathcal{K}$. Notice that $Y(\cdot \mid \Xi)$ can be seen as one possible way of describing a random tessellation in $\Xi$.

Furthermore, if $X=\left\{\Xi_{n}\right\}_{n \geq 1}$ is an arbitrary random tessellation in $\mathbb{R}^{d}$ and if $\left\{X_{n}\right\}_{n \geq 1}$ is a sequence of independent, identically distributed random tessellations $X_{n}=\left\{\Xi_{n v}\right\}_{v \geq 1}$ in $\mathbb{R}^{d}$ (also independent of $X$ ), then the mapping $Y: \Omega \rightarrow N\left(\mathcal{F}^{\prime}\right)$, defined by $Y(B)=$ $\sum_{n \geq 1} Y_{n}\left(B \mid \Xi_{n}\right)$ and $Y_{n}\left(B \mid \Xi_{n}\right)=\sum_{\nu \geq 1} \delta_{\Xi_{n v} \cap \Xi_{n}}(B) \mathbf{1}_{\left\{\text {int } \Xi_{n v} \cap \text { int } \Xi_{n} \neq \varnothing\right\}}$ for $B \in \mathscr{B}\left(\mathcal{F}^{\prime}\right)$, is called the point-process representation of an iterated random tessellation (or the $X / X_{n}$-nesting) in $\mathbb{R}^{d}$ with initial tessellation $X$ and component tessellations $X_{1}, X_{2}, \ldots$ Clearly, the point process $Y$ is stationary and isotropic, provided that the initial tessellation $X$ and the component tessellations $X_{1}, X_{2}, \ldots$ possess these properties. Moreover, $Y$ is ergodic if $X$ is ergodic.

\section{The expectation vector and the covariance matrix}

Let $X=\left\{\Xi_{n}\right\}_{n \geq 1}$ be an arbitrary stationary and ergodic tessellation in $\mathbb{R}^{d}$. Recall that the tessellation $X$ can be equivalently described as a stationary and ergodic marked point process $\sum_{n \geq 1} \delta_{\left[\alpha\left(\Xi_{n}\right), \Xi_{n}^{0}\right]}$, where $\Xi_{n}^{0}=\Xi_{n}-\alpha\left(\Xi_{n}\right)$ and $\alpha\left(\Xi_{n}\right)$ denotes the associated point of $\Xi_{n}$. The intensity $\lambda=\operatorname{Ecard}\left\{n: \alpha\left(\Xi_{n}\right) \in[0,1)^{d}\right\}$ is assumed to be positive and finite. For each individual cell $\Xi_{n}$ of $X$, we consider an $m$-dimensional vector $J_{n}=\left(J_{n}^{(1)}, \ldots, J_{n}^{(m)}\right)^{\top}$ of stationary random measures in $\mathbb{R}^{d}$, which together describe the inner structure of $\Xi_{n}$. We assume that the sequence $\left(J_{n}\right)_{n \geq 1}$ is independent of $X$ and consists of independent, identically distributed copies of a generic stationary random vector measure $J_{0}=\left(J_{0}^{(1)}, \ldots, J_{0}^{(m)}\right)^{\top}$.

Throughout this paper, we assume that only a single realization of the tessellation $X$ and of the random vectors $J_{n}\left(\Xi_{n} \cap W_{\varrho}\right), n \geq 1$, can be observed in an (unboundedly increasing) sampling window $W_{\varrho}=\varrho W \uparrow \mathbb{R}^{d}$ (as $\left.\varrho \uparrow \infty\right)$, where the convex body $W$ satisfies the inclusion $b(o, r) \subseteq W \subseteq b(o, R)$ for some fixed $0<r<R<\infty$. To begin with, we determine the expectation vector $\mathrm{E} Z_{\varrho}$ of the random vector $Z_{\varrho}=\left(Z_{\varrho}^{(1)}, \ldots, Z_{\varrho}^{(m)}\right)^{\top}$ defined by (1.1). Then we derive conditions under which the covariance matrix $\operatorname{cov}\left(Z_{\varrho}\right)$ and the limit $\lim _{\varrho \rightarrow \infty} \operatorname{cov}\left(\widetilde{Z}_{\varrho}\right)$ exist, where $\widetilde{Z}_{\varrho}$ is the normalized vector of functionals, introduced in (1.2).

Theorem 3.1. If $\lambda^{(i)}=\mathrm{E} J_{0}^{(i)}\left([0,1)^{d}\right)<\infty$ for each $i=1, \ldots, m$, then

$$
\mathrm{E} Z_{\varrho}=\left|W_{\varrho}\right|\left(\lambda^{(1)}, \ldots, \lambda^{(m)}\right)^{\top} .
$$


Under the additional assumption that

$$
\int_{\mathcal{P}^{0}} \mathrm{E}\left(J_{0}^{(i)}(C)\right)^{2} P^{0}(\mathrm{~d} C)<\infty, \quad i=1, \ldots, m,
$$

the covariance matrix $\operatorname{cov}\left(Z_{\varrho}\right)=\left(\operatorname{cov}\left(Z_{\varrho}^{(i)}, Z_{\varrho}^{(j)}\right)\right)_{i, j=1}^{m}$ exists with entries taking the form

$$
\operatorname{cov}\left(Z_{\varrho}^{(i)}, Z_{\varrho}^{(j)}\right)=\lambda \int_{\mathcal{P}^{0}} \int_{\mathbb{R}^{d}} \operatorname{cov}\left(J_{0}^{(i)}\left(C \cap\left(W_{\varrho}-x\right)\right), J_{0}^{(j)}\left(C \cap\left(W_{\varrho}-x\right)\right)\right) \mathrm{d} x P^{0}(\mathrm{~d} C) .
$$

Moreover, the asymptotic covariance matrix $K=\lim _{\varrho \rightarrow \infty} \operatorname{cov}\left(\widetilde{Z}_{\varrho}\right)=\left(\sigma_{i j}^{2}\right)_{i, j=1}^{m}$ exists, and has entries

$$
\sigma_{i j}^{2}=\lambda \int_{\mathcal{P}^{0}} \operatorname{cov}\left(J_{0}^{(i)}(C), J_{0}^{(j)}(C)\right) P^{0}(\mathrm{~d} C), \quad i, j=1, \ldots, m .
$$

Proof. In view of the independence of $X$ and the sequence $\left(J_{n}\right)_{n \geq 1}$ we may write, for any $i=1, \ldots, m$, that

$$
\mathrm{E} Z_{\varrho}^{(i)}=\mathrm{E}\left(\sum_{n \geq 1} J_{n}^{(i)}\left(\Xi_{n} \cap W_{\varrho}\right)\right)=\mathrm{E}\left(\sum_{n \geq 1} \mathrm{E}_{X}\left(J_{0}^{(i)}\left(\Xi_{n} \cap W_{\varrho}\right)\right)\right)
$$

where $\mathrm{E}_{X}$ denotes the conditional expectation, given the tessellation $X$. By the stationarity of $J_{0}^{(i)}$, the expectation $\mathrm{E}_{X}\left(J_{0}^{(i)}\left(\Xi_{n} \cap W_{\varrho}\right)\right)$ equals $\lambda^{(i)}\left|\Xi_{n} \cap W_{\varrho}\right|$. Since the interior of the cells $\Xi_{n}, n \geq 1$, fills the space $\mathbb{R}^{d}$ up to a set of Lebesgue measure 0 , we have

$$
\sum_{n \geq 1}\left|\Xi_{n} \cap W_{\varrho}\right|=\left|W_{\varrho}\right|
$$

which proves (3.1). To derive (3.3), we will first carry out the necessary calculations without regard to the existence of the integrals and expectations involved, and after that we will check their validity. Once more using the independence of $X$ and the sequence $\left(J_{n}\right)_{n \geq 1}$, and combining these with (3.1), we get

$$
\begin{aligned}
\operatorname{cov}\left(Z_{\varrho}^{(i)}, Z_{\varrho}^{(j)}\right)= & \mathrm{E}\left(\sum_{n \geq 1} \mathrm{E}_{X}\left(J_{0}^{(i)}\left(\Xi_{n} \cap W_{\varrho}\right) J_{0}^{(j)}\left(\Xi_{n} \cap W_{\varrho}\right)\right)\right) \\
& +\mathrm{E}\left(\sum_{\substack{n, \ell \geq 1 \\
n \neq \ell}} \mathrm{E}_{X}\left(J_{n}^{(i)}\left(\Xi_{n} \cap W_{\varrho}\right) J_{\ell}^{(j)}\left(\Xi_{\ell} \cap W_{\varrho}\right)\right)\right)-\lambda^{(i)} \lambda^{(j)}\left|W_{\varrho}\right|^{2} .
\end{aligned}
$$

The difference of the two expressions in the second line is

$$
-\mathrm{E}\left(\sum_{n \geq 1} \mathrm{E}_{X}\left(J_{0}^{(i)}\left(\Xi_{n} \cap W_{\varrho}\right)\right) \mathrm{E}_{X}\left(J_{0}^{(j)}\left(\Xi_{n} \cap W_{\varrho}\right)\right)\right)
$$

since, for $n \neq \ell$, we have

$$
\mathrm{E}_{X}\left(J_{n}^{(i)}\left(\Xi_{n} \cap W_{\varrho}\right) J_{\ell}^{(j)}\left(\Xi_{\ell} \cap W_{\varrho}\right)\right)=\mathrm{E}_{X} J_{n}^{(i)}\left(\Xi_{n} \cap W_{\varrho}\right) \mathrm{E}_{X} J_{\ell}^{(j)}\left(\Xi_{\ell} \cap W_{\varrho}\right)
$$


by the assumed independence of $J_{n}^{(i)}$ and $J_{\ell}^{(i)}$. Thus, denoting by $\operatorname{cov}_{X}$ the conditional covariance given the tessellation $X=\left\{\Xi_{n}\right\}_{n \geq 1}$, we find that

$$
\operatorname{cov}\left(Z_{\varrho}^{(i)}, Z_{\varrho}^{(j)}\right)=\mathrm{E}\left(\sum_{n \geq 1} \operatorname{cov}_{X}\left(J_{0}^{(i)}\left(\Xi_{n} \cap W_{\varrho}\right), J_{0}^{(j)}\left(\Xi_{n} \cap W_{\varrho}\right)\right)\right) .
$$

Finally, writing $\Xi_{n}=\Xi_{n}^{0}+\alpha\left(\Xi_{n}\right)$ and applying Campbell's theorem to the stationary marked point process $\sum_{n \geq 1} \delta_{\left[\alpha\left(\Xi_{n}\right), \Xi_{n}^{0}\right]}$, we get

$$
\begin{aligned}
\operatorname{cov}\left(Z_{\varrho}^{(i)}, Z_{\varrho}^{(j)}\right) & =\lambda \int_{\mathbb{R}^{d}} \int_{\mathcal{P}^{0}} \operatorname{cov}\left(J_{0}^{(i)}\left((C+x) \cap W_{\varrho}\right), J_{0}^{(j)}\left((C+x) \cap W_{\varrho}\right)\right) P^{0}(\mathrm{~d} C) \mathrm{d} x \\
& =\lambda \int_{\mathcal{P}^{0}} \int_{\mathbb{R}^{d}} \operatorname{cov}\left(J_{0}^{(i)}\left(C \cap\left(W_{\varrho}-x\right)\right), J_{0}^{(j)}\left(C \cap\left(W_{\varrho}-x\right)\right)\right) \mathrm{d} x P^{0}(\mathrm{~d} C),
\end{aligned}
$$

where, in the second equality, we have used Fubini's theorem and the invariance of $\operatorname{cov}\left(J_{0}^{(i)}(A)\right.$, $\left.J_{0}^{(j)}(B)\right)$ under diagonal shifts, i.e.

$$
\operatorname{cov}\left(J_{0}^{(i)}(A+x), J_{0}^{(j)}(B+x)\right)=\operatorname{cov}\left(J_{0}^{(i)}(A), J_{0}^{(j)}(B)\right)
$$

for any $A, B \in \mathcal{C}$ and for any $x \in \mathbb{R}^{d}$.

To complete the proof of (3.3), we justify the steps and changes of integration above by showing that our integrability condition (3.2) ensures the existence of the second moment

$$
\mathrm{E} \sum_{n \geq 1}\left(J_{n}^{(i)}\left(\Xi_{n} \cap W_{\varrho}\right)\right)^{2}=\lambda \int_{\mathcal{P}^{0}} \int_{\mathbb{R}^{d}} \mathrm{E}\left(J_{0}^{(i)}\left(C \cap\left(W_{\varrho}-x\right)\right)\right)^{2} \mathrm{~d} x P^{0}(\mathrm{~d} C)
$$

for each $i=1, \ldots, m$. By Fubini's theorem, we have

$$
\begin{aligned}
\int_{\mathbb{R}^{d}}\left(J_{0}^{(i)}\left(C \cap\left(W_{\varrho}-x\right)\right)\right)^{2} \mathrm{~d} x & =\int_{C} \int_{C}\left|\left(W_{\varrho}-y\right) \cap\left(W_{\varrho}-z\right)\right| J_{0}^{(i)}(\mathrm{d} y) J_{0}^{(i)}(\mathrm{d} z) \\
& \leq\left|W_{\varrho}\right|\left(J_{0}^{(i)}(C)\right)^{2}
\end{aligned}
$$

and, therefore, by means of (3.2),

$$
\mathrm{E} \sum_{n \geq 1}\left(J_{n}^{(i)}\left(\Xi_{n} \cap W_{\varrho}\right)\right)^{2} \leq \lambda\left|W_{\varrho}\right| \int_{\mathcal{P}^{0}} \mathrm{E}\left(J_{0}^{(i)}(C)\right)^{2} P^{0}(\mathrm{~d} C)<\infty
$$

for $i=1, \ldots, m$. The existence of the other expressions can be demonstrated using the Cauchy-Schwarz inequality, which proves (3.3). In order to show (3.4), notice that the properties of the family of convex sets $\left(W_{\varrho}\right)_{\varrho>0}$ imply that

$$
\lim _{\varrho \rightarrow \infty} \frac{\left|\left(W_{\varrho}-y\right) \cap\left(W_{\varrho}-z\right)\right|}{\left|W_{\varrho}\right|}=1 \text { for any fixed } y, z \in \mathbb{R}^{d} .
$$

Hence, bounding the mixed second moments $\operatorname{E}\left(J_{0}^{(i)}\left(C \cap\left(W_{\varrho}-x\right)\right) J_{0}^{(j)}\left(C \cap\left(W_{\varrho}-x\right)\right)\right)$ from above - quite similarly to as was done in (3.6) - and using the dominated convergence theorem, we obtain

$$
\lim _{\varrho \rightarrow \infty} \int_{\mathbb{R}^{d}} \frac{\mathrm{E}\left(J_{0}^{(i)}\left(C \cap\left(W_{\varrho}-x\right)\right) J_{0}^{(j)}\left(C \cap\left(W_{\varrho}-x\right)\right)\right)}{\left|W_{\varrho}\right|} \mathrm{d} x=\mathrm{E}\left(J_{0}^{(i)}(C) J_{0}^{(j)}(C)\right)
$$


for any $C \in \mathcal{P}^{0}$ and $i, j=1, \ldots, m$. The previous relation remains true for the first-order moments resulting from the covariance formula (3.5). Thus, applying the theorems of Campbell and Fubini to (3.5), together with the dominated convergence theorem, we finally obtain that

$$
\begin{aligned}
& \lim _{\varrho \rightarrow \infty} \frac{\operatorname{cov}\left(Z_{\varrho}^{(i)}, Z_{\varrho}^{(j)}\right)}{\left|W_{\varrho}\right|} \\
& \quad=\lambda \int_{\mathcal{P}^{0}} \lim _{\varrho \rightarrow \infty} \int_{\mathbb{R}^{d}} \frac{\operatorname{cov}\left(J_{0}^{(i)}\left(C \cap\left(W_{\varrho}-x\right)\right), J_{0}^{(j)}\left(C \cap\left(W_{\varrho}-x\right)\right)\right)}{\left|W_{\varrho}\right|} \mathrm{d} x P^{0}(\mathrm{~d} C)
\end{aligned}
$$

for any $i, j=1, \ldots, m$, where the expression on the right-hand side coincides with $\sigma_{i j}^{2}$, as was defined in (3.4).

Note that the second part of Theorem 3.1 implies that the asymptotic variance of the scalar product $t^{\top} \widetilde{Z}_{\varrho}=\sum_{l=1}^{m} t_{l} \widetilde{Z}_{\varrho}^{(l)}$ exists for any $t=\left(t_{1}, \ldots, t_{m}\right)^{\top} \in \mathbb{R}^{m} ;$ more precisely,

$$
\lim _{\varrho \rightarrow \infty} \operatorname{var}\left(t^{\top} \widetilde{Z}_{\varrho}\right)=t^{\top} K t=\sum_{i, j=1}^{m} t_{i} t_{j} \sigma_{i j}^{2},
$$

where $K=\left(\sigma_{i j}^{2}\right)_{i, j=1}^{m}$ is given by (3.4).

We conclude this section with a discussion of the integrability conditions in (3.2). Our aim is to put separate conditions on the random measures $J_{0}^{(i)}$ and the typical cell $\Xi^{*}$ of $X$, which together imply (3.2).

Lemma 3.1. The inequalities

$$
\left(\lambda^{(i)}\right)^{2} \mathrm{E}\left|\Xi^{*}\right|^{2} \leq \int_{\mathcal{P}^{0}} \mathrm{E}\left(J_{0}^{(i)}(C)\right)^{2} P^{0}(\mathrm{~d} C) \leq \mathrm{E}\left|\Xi^{*} \oplus b(o, \sqrt{d})\right|^{2} \mathrm{E}\left(J_{0}^{(i)}\left([0,1)^{d}\right)\right)^{2}
$$

hold for each $i=1, \ldots, m$. Consequently, (3.2) is satisfied whenever

$$
\mathrm{E} M_{k}^{2}\left(\Xi^{*}\right)<\infty \text { and } \mathrm{E}\left(J_{0}^{(i)}\left([0,1)^{d}\right)\right)^{2}<\infty
$$

for any $k=0, \ldots, d-1$ and $i=1, \ldots, m$.

Proof. Taking into account the fact that $\mathrm{E} J_{0}^{(i)}(C)=\lambda^{(i)}|C|$, the first inequality in (3.8) immediately follows from Jensen's inequality, namely $\left(\mathrm{E} J_{0}^{(i)}(C)\right)^{2} \leq \mathrm{E}\left(J_{0}^{(i)}(C)\right)^{2}$. The obvious set-theoretic inclusions

$$
C \subseteq \bigcup_{\left\{z \in \mathbb{Z}^{d}: C \cap\left([0,1)^{d}+z\right) \neq \varnothing\right\}}\left([0,1)^{d}+z\right) \subseteq C \oplus b(o, \sqrt{d}),
$$

which are true for any subset $C$ of $\mathbb{R}^{d}$, and Steiner's formula (2.1) imply that

$$
\operatorname{card}\left\{z \in \mathbb{Z}^{d}: C \cap\left([0,1)^{d}+z\right) \neq \varnothing\right\} \leq|C \oplus b(o, \sqrt{d})|=\sum_{k=0}^{d}\left(\begin{array}{l}
d \\
k
\end{array}\right) M_{k}(C) d^{k / 2}
$$

for each $C \in \mathcal{C}$. Thus, using the monotonicity and translation invariance of the set function $\mathrm{E}\left(J_{0}^{(i)}(\cdot)\right)^{2}$, and the elementary inequality $\left(a_{1}+\cdots+a_{N}\right)^{2} \leq N\left(a_{1}^{2}+\cdots+a_{N}^{2}\right)$, for any 
integer $N>0$, we arrive at

$$
\begin{aligned}
\mathrm{E}\left(J_{0}^{(i)}(C)\right)^{2} & \leq \operatorname{card}\left\{z \in \mathbb{Z}_{C}^{d}\right\} \sum_{z \in \mathbb{Z}_{C}^{d}} \mathrm{E}\left(J_{0}^{(i)}\left([0,1)^{d}+z\right)\right)^{2} \\
& \leq|C \oplus b(o, \sqrt{d})|^{2} \mathrm{E}\left(J_{0}^{(i)}\left([0,1)^{d}\right)\right)^{2}
\end{aligned}
$$

where $\mathbb{Z}_{C}^{d}=\left\{z \in \mathbb{Z}^{d}: C \cap\left([0,1)^{d}+z\right) \neq \varnothing\right\}$. This proves the second inequality in (3.8). Furthermore, by (3.10), $\mathrm{E}\left|\Xi^{*} \oplus b\left(o, d^{1 / 2}\right)\right|^{2}<\infty$ if the first integrability condition in (3.9) is satisfied.

In some cases, it might not be possible to directly check whether or not the first integrability condition in (3.9) is satisfied. This is due to the fact that it is sometimes difficult to determine the second moment $\mathrm{E} M_{k}^{2}\left(\Xi^{*}\right)$ of the $k$ th Minkowski-functional $M_{k}\left(\Xi^{*}\right)$ of the typical cell $\Xi^{*}$ of $X$. However, the isodiametric inequality (2.2) implies that $\mathrm{E} M_{k}^{2}\left(\Xi^{*}\right)<\infty$ holds for each $k=0, \ldots, d-1$, provided that

$$
\mathrm{E} D^{2 d}\left(\Xi^{*}\right)<\infty
$$

\section{Laws of large numbers}

Recall that the individual ergodic theorem applied to the (stationary and ergodic) marked point process $\sum_{n \geq 1} \delta_{\left[\alpha\left(\Xi_{n}\right), \Xi_{n}^{0}\right]}$ reads as follows (see [6, p. 339]): for any real-valued integrable function $h$ defined on the probability space $\left(\mathcal{P}^{0}, \mathcal{B}(\mathcal{F}) \cap \mathcal{P}^{0}, P^{0}\right)$, we have

$$
\frac{1}{\left|W_{\varrho}\right|} \sum_{n \geq 1} \mathbf{1}_{W_{\varrho}}\left(\alpha\left(\Xi_{n}\right)\right) h\left(\Xi_{n}^{0}\right) \underset{\varrho \rightarrow \infty}{\stackrel{\text { a.s. }}{\longrightarrow}} \lambda \mathrm{E} h\left(\Xi^{*}\right)=\lambda \int_{\mathcal{P}^{0}} h(C) P^{0}(\mathrm{~d} C) .
$$

However, in the context of this paper, as in many other statistical applications of the spatial ergodic theorem (4.1), we have to consider spatial averages over cells of $X=\left\{\Xi_{n}\right\}_{n \geq 1}$ that only partly belong to the sampling window $W_{\varrho}$. Such boundary effects are taken into account in the following result, which asserts the strong consistency of a (not necessarily unbiased) estimator for $\lambda \mathrm{E} g\left(\Xi^{*}\right)$ in the case of a nonrandom, translation-invariant, and isotonic functional on $\mathcal{C}$.

Theorem 4.1. Let $g: \mathcal{C} \rightarrow[0, \infty)$ be a $\mathcal{B}(\mathcal{F})$-measurable, nonnegative set function such that $g(C) \leq g\left(C^{\prime}\right)$ for $C \subseteq C^{\prime}$ and $g(C)=g(C+x)$ for any $C \in \mathcal{C}$ and $x \in R^{d}$. If the typical cell $\Xi^{*}$ of $X$ satisfies

$$
\mathrm{E} D^{d}\left(\Xi^{*}\right)<\infty \text { and } \mathrm{E} g\left(\Xi^{*}\right)<\infty
$$

then

$$
\frac{1}{\left|W_{\varrho}\right|} \sum_{n \geq 1} \mathbf{1}_{\left\{\Xi_{n} \cap W_{\varrho} \neq \varnothing\right\}} g\left(\Xi_{n} \cap W_{\varrho}\right) \underset{\varrho \rightarrow \infty}{\stackrel{\text { a.s. }}{\longrightarrow}} \lambda \mathrm{E} g\left(\Xi^{*}\right) .
$$

The proof of Theorem 4.1 is postponed to Section 4.2. Notice, however, that Theorem 4.1 is not completely new. For instance, in the planar case $d=2$, one can find it in [5] applied to some particular functionals $g\left(\Xi_{n} \cap W_{\varrho}\right)$ of the cells $\Xi_{n} \cap W_{\varrho}$, whereas we consider a general class of isotonic and translation-invariant functionals $g: \mathcal{C} \rightarrow[0, \infty)$. Furthermore, a straightforward application of Theorem 4.1 to each of the particular functionals

$$
g_{1}(C)=\mathrm{E}\left(J_{0}^{(i)}(C) J_{0}^{(j)}(C)\right) \quad \text { and } \quad g_{2}(C)=|C|^{2}, \quad 1 \leq i, j \leq m, C \in \mathcal{C},
$$

yields the following result. 
Corollary 4.1. Let $J_{0}=\left(J_{0}^{(1)}, \ldots, J_{0}^{(m)}\right)^{\top}$ be a vector of stationary random measures on $\mathbb{R}^{d}$, which are independent of $X$ and satisfy both (3.2) (or (3.9)) and $\mathrm{E} D^{d}\left(\Xi^{*}\right)<\infty$. Then, for any $t=\left(t_{1}, \ldots, t_{m}\right)^{\top} \in \mathbb{R}^{m}$,

$$
\frac{1}{\left|W_{\varrho}\right|} \sum_{n \geq 1} \mathrm{E}_{X}\left(t^{\top} J_{0}\left(\Xi_{n} \cap W_{\varrho}\right)\right)^{2} \underset{\varrho \rightarrow \infty}{\stackrel{\text { a.s. }}{\longrightarrow}} \lambda \mathrm{E}\left(t^{\top} J_{0}\left(\Xi^{*}\right)\right)^{2}
$$

and

$$
\frac{1}{\left|W_{\varrho}\right|} \sum_{n \geq 1}\left|\Xi_{n} \cap W_{\varrho}\right|^{2} \underset{\varrho \rightarrow \infty}{\stackrel{\text { a.s. }}{\longrightarrow}} \lambda \mathrm{E}\left|\Xi^{*}\right|^{2} .
$$

It transpires that the result of Theorem 4.1 remains true if the (nonrandom) functional $g: \mathcal{C} \rightarrow[0, \infty)$ is replaced by the stationary random measures $J_{n}^{(i)}$.

Theorem 4.2. Assume that $\mathrm{E} D^{d}\left(\Xi^{*}\right)<\infty$ and $\lambda^{(i)}=\mathrm{E} J_{0}^{(i)}\left([0,1)^{d}\right)<\infty$ for $i=1, \ldots, m$. Then,

$$
\frac{1}{\left|W_{\varrho}\right|} \sum_{n \geq 1} J_{n}^{(i)}\left(\Xi_{n} \cap W_{\varrho}\right) \underset{\varrho \rightarrow \infty}{\stackrel{\text { a.s. }}{\longrightarrow}} \lambda^{(i)}, \quad i=1, \ldots, m .
$$

The proof of Theorem 4.2 will be given in Section 4.3, below. Notice that the limit $\lambda^{(i)}$ in (4.5) corresponds to that of (4.2) because the stationarity of $J_{0}^{(i)}$ and (2.3) imply that $\lambda^{(i)}=\lambda \mathrm{E} J_{0}^{(i)}\left(\Xi^{*}\right)$, provided that $J_{0}$ and $\Xi^{*}$ are independent.

\subsection{Cells hitting the boundary of the sampling window}

The following lemma is essential for the proof of Theorem 4.1. However, it also seems to be of interest in its own right: we show that the influence of those cells $\Xi_{n}$ of $X$ (appearing on the left-hand side of (4.2)) that hit the boundary $\partial W_{\varrho}$ becomes asymptotically negligible as $\varrho \rightarrow \infty$. To do so, we define the families of events $\left(A_{\varrho}\right)_{\varrho>\varrho_{0}}$ and $\left(B_{\varrho}\right)_{\varrho>\varrho_{0}}$ by

$$
A_{\varrho}=\bigcap_{n \geq 1}\left(\left\{\left(\Xi_{n}^{0}+\alpha\left(\Xi_{n}\right)\right) \cap W_{\varrho}=\varnothing\right\} \cup\left\{\alpha\left(\Xi_{n}\right) \in W_{\varrho+q(\varrho)}\right\}\right)
$$

and

$$
B_{\varrho}=\bigcap_{n \geq 1}\left(\left\{\left(\Xi_{n}^{0}+\alpha\left(\Xi_{n}\right)\right) \subseteq W_{\varrho}\right\} \cup\left\{\alpha\left(\Xi_{n}\right) \notin W_{\varrho-q(\varrho)}\right\}\right),
$$

where $\varrho_{0} \geq 0$ is some constant and the function $q:\left(\varrho_{0}, \infty\right) \rightarrow(0, \infty)$ is such that $q(\varrho)<\varrho$ for each $\varrho>\varrho_{0}$.

Lemma 4.1. Under the assumption that $\mathrm{E} D^{d}\left(\Xi^{*}\right)<\infty$, there exists a nondecreasing function $q:\left(\varrho_{0}, \infty\right) \rightarrow(0, \infty)$ satisfying $q(\varrho)<\varrho$ for $\varrho>\varrho_{0}, q(\varrho) \rightarrow \infty$, and $q(\varrho) / \varrho \downarrow 0$ as $\varrho \rightarrow \infty$, such that

$$
\lim _{\ell \rightarrow \infty} \mathrm{P}\left(\bigcup_{k \geq \ell} A_{k}^{\mathrm{c}}\right)=0 \text { and } \lim _{\ell \rightarrow \infty} \mathrm{P}\left(\bigcup_{k \geq \ell} B_{k}^{\mathrm{c}}\right)=0
$$

Proof. To begin, we recall the well-known fact from analysis that the integrability of $D^{d}\left(\Xi^{*}\right)$ implies the existence of a convex function $H:[0, \infty) \rightarrow[0, \infty)$, strictly increasing on its support $\left(x_{0}, \infty\right)$ (for some $x_{0} \geq 0$ ), such that $H(x) / x$ is nondecreasing for $x>0$, $\lim _{x \rightarrow \infty} H(x) / x=\infty$, and E $H\left(D^{d}\left(\Xi^{*}\right)\right)<\infty$ (see, e.g. Theorem II.22 of [7]). Furthermore, 
for some $r>0$ such that $b(o, r) \subseteq W$, the value $q(\varrho)$ - greater than $x_{0}^{1 / d} / r-$ is defined as the unique solution of the equation

$$
\varrho^{d}=H\left(r^{d} q^{d}(\varrho)\right) \text { for any } \varrho>0 .
$$

It is easily checked that the function $\varrho \mapsto q(\varrho)$ possesses the properties required for $\varrho>\varrho_{0}=$ $\inf \left\{x>0: H\left(r^{d} q^{d}(x)\right)>q^{d}(x)\right\}$ to hold. Note that $b(o, r) \subseteq W$ implies that $b(o, r q(\varrho)) \subseteq$ $W_{q(\varrho)}$ and, by the convexity of $W$, that

$$
W_{\varrho} \oplus b(o, r q(\varrho)) \subseteq W_{\varrho} \oplus W_{q(\varrho)} \subseteq W_{\varrho+q(\varrho)} .
$$

Thus, for any $C \in \mathcal{C}$ with $o \in C$, we have $D(C)>r q(\varrho)$, provided that $W_{\varrho+q(\varrho)}^{\mathrm{c}} \cap\left(W_{\varrho} \oplus C\right) \neq$ $\varnothing$. Together with the definition of $A_{\varrho}$, this yields that

$$
\begin{aligned}
\mathrm{P}\left(\bigcup_{k \geq \ell} A_{k}^{\mathrm{c}}\right) & =\mathrm{P}\left(\bigcup_{n \geq 1} \bigcup_{k \geq \ell}\left\{\alpha\left(\Xi_{n}\right) \in W_{k+q(k)}^{\mathrm{c}} \cap\left(W_{k} \oplus \check{\Xi}_{n}^{0}\right)\right\}\right) \\
& \leq \mathrm{P}\left(\bigcup_{n \geq 1} \bigcup_{k \geq \ell}\left(\left\{\alpha\left(\Xi_{n}\right) \in W_{k} \oplus b\left(o, D\left(\Xi_{n}^{0}\right)\right)\right\} \cap\left\{D\left(\Xi_{n}^{0}\right)>r q(k)\right\}\right)\right) \\
& =\mathrm{P}\left(\bigcup_{n \geq 1} \bigcup_{k \geq \ell}\left(\left\{\alpha\left(\Xi_{n}\right) \in W_{k} \oplus b\left(o, D\left(\Xi_{n}^{0}\right)\right)\right\} \cap\left\{r q(k)<D\left(\Xi_{n}^{0}\right) \leq r q(k+1)\right\}\right)\right) .
\end{aligned}
$$

The last equality follows from the fact that, for any two sequences of events $\left\{E_{k}\right\}_{k \geq \ell}$ and $\left\{E_{k}^{\prime}\right\}_{k \geq \ell}$ with $E_{k} \subseteq E_{k+1}$ and $E_{k}^{\prime} \supseteq E_{k+1}^{\prime}$ for $k \geq \ell$, the identity

$$
\bigcup_{k \geq \ell} E_{k} \cap E_{k}^{\prime}=\bigcup_{k \geq \ell} E_{k} \cap\left(E_{k}^{\prime} \backslash E_{k+1}^{\prime}\right)
$$

holds. Thus, the subadditivity of $\mathrm{P}$, the inequality $\mathrm{P}(U \geq 1) \leq \mathrm{E}|U|$ and Campbell's theorem for stationary marked point processes imply that

$$
\begin{aligned}
\mathrm{P}\left(\bigcup_{k \geq \ell} A_{k}^{\mathrm{c}}\right) & \leq \sum_{k \geq \ell} \mathrm{P}\left(\sum_{n \geq 1} \mathbf{1}_{W_{k} \oplus b\left(o, D\left(\Xi_{n}^{0}\right)\right)}\left(\alpha\left(\Xi_{n}\right)\right) \mathbf{1}_{(r q(k), r q(k+1)]}\left(D\left(\Xi_{n}^{0}\right)\right) \geq 1\right) \\
& \leq \sum_{k \geq \ell} \mathrm{E}\left(\sum_{n \geq 1} \mathbf{1}_{W_{k} \oplus b\left(o, D\left(\Xi_{n}^{0}\right)\right)}\left(\alpha\left(\Xi_{n}\right)\right) \mathbf{1}_{(r q(k), r q(k+1)]}\left(D\left(\Xi_{n}^{0}\right)\right)\right) \\
& =\lambda \sum_{k \geq \ell} \int_{\mathcal{P}^{0}}\left|W_{k} \oplus b(o, D(C))\right| \mathbf{1}_{(r q(k), r q(k+1)]}(D(C)) P^{0}(\mathrm{~d} C) .
\end{aligned}
$$

Since $W_{k}=k W \in \mathcal{C}$, we now are in a position to apply Steiner's formula (2.1), which, together with the homogeneity relation $M_{S}\left(W_{k}\right)=k^{d-s} M_{S}(W)$ for the Minkowski-functionals $M_{s}$ (see, e.g. [11]) and the monotonicity of the function $H(\cdot)$, leads to

$$
\begin{aligned}
\mathrm{P}\left(\bigcup_{k \geq \ell} A_{k}^{\mathrm{c}}\right) & \leq \lambda \sum_{k \geq \ell} \sum_{s=0}^{d}\left(\begin{array}{l}
d \\
s
\end{array}\right) k^{d-s} M_{S}(W) \mathrm{E}\left(D^{s}\left(\Xi^{*}\right) \mathbf{1}_{(r q(k), r q(k+1)]}\left(D\left(\Xi^{*}\right)\right)\right) \\
& \leq \lambda \sum_{k \geq \ell} \sum_{s=0}^{d}\left(\begin{array}{l}
d \\
s
\end{array}\right) M_{s}(W) \frac{k^{d-s} r^{s} q^{s}(k+1)}{H\left(r^{d} q^{d}(k)\right)} \mathrm{E}\left(H\left(D^{d}\left(\Xi^{*}\right)\right) \mathbf{1}_{(r q(k), r q(k+1)]}\left(D\left(\Xi^{*}\right)\right)\right) .
\end{aligned}
$$


By (4.9), we have $H\left(r^{d} q^{d}(k)\right)=k^{d}$ and $q(k+1) \leq k+1$ for all $k \geq \ell$ (with $\ell$ large enough) and, therefore,

$$
\frac{k^{d-s} r^{s} q^{s}(k+1)}{H\left(r^{d} q^{d}(k)\right)} \leq\left(1+\frac{1}{k}\right)^{s} r^{s} \leq 2^{s} r^{s} \quad \text { for } k \geq \ell
$$

This yields

$$
\mathrm{P}\left(\bigcup_{k \geq \ell} A_{k}^{\mathrm{c}}\right) \leq \lambda \sum_{s=0}^{d}\left(\begin{array}{l}
d \\
s
\end{array}\right)(2 r)^{s} M_{s}(W) \mathrm{E}\left(H\left(D^{d}\left(\Xi^{*}\right)\right) \mathbf{1}_{(r q(\ell), \infty)}\left(D\left(\Xi^{*}\right)\right)\right) \underset{\ell \rightarrow \infty}{\longrightarrow} 0 .
$$

To show the second assertion in (4.8) we first note that

$$
\mathrm{P}\left(\bigcup_{k \geq \ell} B_{k}^{\mathrm{c}}\right)=\mathrm{P}\left(\bigcup_{n \geq 1} \bigcup_{k \geq \ell}\left\{\alpha\left(\Xi_{n}\right) \in W_{k-q(k)} \cap\left(W_{k}^{\mathrm{c}} \oplus \check{\Xi}_{n}^{0}\right)\right\}\right) .
$$

In analogy to the considerations above, one can show that, for any $C \in \mathcal{C}$ with $o \in C$, we have $D(C)>r q(\varrho)$, provided that $\left(W_{\varrho}^{\mathrm{c}} \oplus C\right) \cap W_{\varrho-q(\varrho)} \neq \varnothing$. Together with $W_{k-q(k)} \subseteq W_{k}$, this implies that

$$
\mathrm{P}\left(\bigcup_{k \geq \ell} B_{k}^{\mathrm{c}}\right) \leq \mathrm{P}\left(\bigcup_{n \geq 1} \bigcup_{k \geq \ell}\left(\left\{\alpha\left(\Xi_{n}\right) \in W_{k}\right\} \cap\left\{D\left(\Xi_{n}^{0}\right)>r q(k)\right\}\right)\right) .
$$

Since the right-hand side of this inequality is no larger than the bound on the right-hand side of (4.10), we immediately find that $\mathrm{P}\left(\bigcup_{k \geq \ell} B_{k}^{\mathrm{c}}\right) \rightarrow 0$ as $\ell \rightarrow \infty$.

\subsection{Proof of Theorem 4.1}

For notational simplicity, we let

$$
\Delta_{k}=\frac{1}{\left|W_{k}\right|} \sum_{n \geq 1} \mathbf{1}_{\left\{\left(\Xi_{n}^{0}+\alpha\left(\Xi_{n}\right)\right) \cap W_{k} \neq \varnothing\right\}} g\left(\left(\Xi_{n}^{0}+\alpha\left(\Xi_{n}\right)\right) \cap W_{k}\right) \quad \text { for } k \geq \ell .
$$

By rewriting the almost-sure convergence, the assertion of Theorem 4.1 is equivalent to

$$
\mathrm{P}\left(\sup _{k \geq \ell}\left|\Delta_{k}-\lambda \mathrm{E} g\left(\Xi^{*}\right)\right| \geq \delta\right)=\mathrm{P}\left(\bigcup_{k \geq \ell}\left\{\left|\Delta_{k}-\lambda \mathrm{E} g\left(\Xi^{*}\right)\right| \geq \delta\right\}\right) \underset{\ell \rightarrow \infty}{\longrightarrow} 0
$$

for any $\delta>0$ (see, e.g. Lemma 6.8 of [19]). Furthermore,

$$
\begin{aligned}
\mathrm{P}\left(\bigcup_{k \geq \ell}\left\{\left|\Delta_{k}-\lambda \mathrm{E} g\left(\Xi^{*}\right)\right| \geq \delta\right\}\right) \leq & \mathrm{P}\left(\bigcup_{k \geq \ell}\left(\left\{\left|\Delta_{k}-\lambda \mathrm{E} g\left(\Xi^{*}\right)\right| \geq \delta\right\} \cap A_{k} \cap B_{k}\right)\right) \\
& +\mathrm{P}\left(\bigcup_{k \geq \ell}\left(\left\{\left|\Delta_{k}-\lambda \mathrm{E} g\left(\Xi^{*}\right)\right| \geq \delta\right\} \cap\left(A_{k} \cap B_{k}\right)^{\mathrm{c}}\right)\right) \\
\leq & \mathrm{P}\left(\bigcup_{k \geq \ell}\left(\left\{\Delta_{k} \geq \lambda \mathrm{E} g\left(\Xi^{*}\right)+\delta\right\} \cap A_{k}\right)\right)+\mathrm{P}\left(\bigcup_{k \geq \ell} A_{k}^{\mathrm{c}}\right) \\
& +\mathrm{P}\left(\bigcup_{k \geq \ell}\left(\left\{\Delta_{k} \leq \lambda \mathrm{E} g\left(\Xi^{*}\right)-\delta\right\} \cap B_{k}\right)\right)+\mathrm{P}\left(\bigcup_{k \geq \ell} B_{k}^{\mathrm{c}}\right),
\end{aligned}
$$


where the events $A_{k}$ and $B_{k}$ were defined in (4.6) and (4.7), respectively. Taking into account the properties of the functional $g: \mathcal{C} \rightarrow[0, \infty)$ on $A_{k}$ (contained in the assertion of Theorem 4.1), we can verify the inequality

$$
\Delta_{k} \leq \frac{1}{\left|W_{k}\right|} \sum_{n \geq 1} \mathbf{1}_{W_{k+q(k)}}\left(\alpha\left(\Xi_{n}\right)\right) g\left(\Xi_{n}^{0}\right)
$$

for each $k \geq \ell$ with $\ell$ sufficiently large. Likewise, for $k \geq \ell$, we have on $B_{k}$ that

$$
\Delta_{k} \geq \frac{1}{\left|W_{k}\right|} \sum_{n \geq 1} \mathbf{1}_{W_{k-q(k)}}\left(\alpha\left(\Xi_{n}\right)\right) g\left(\Xi_{n}^{0}\right) .
$$

Hence,

$$
\begin{gathered}
\mathrm{P}\left(\bigcup_{k \geq \ell}\left(\left\{\Delta_{k} \geq \lambda_{0} \operatorname{E} g\left(\Xi^{*}\right)+\delta\right\} \cap A_{k}\right)\right)+\mathrm{P}\left(\bigcup_{k \geq \ell}\left(\left\{\Delta_{k} \leq \lambda \operatorname{Eg}\left(\Xi^{*}\right)-\delta\right\} \cap B_{k}\right)\right) \\
\leq \sum_{\kappa \in\{-1,+1\}} \mathrm{P}\left(\sup _{k \geq \ell}\left|\frac{1}{\left|W_{k}\right|} \sum_{n \geq 1} \mathbf{1}_{W_{k+\kappa q(k)}}\left(\alpha\left(\Xi_{n}\right)\right) g\left(\Xi_{n}^{0}\right)-\lambda \mathrm{E} g\left(\Xi^{*}\right)\right| \geq \delta\right) .
\end{gathered}
$$

However, this summation tends to 0 as $\ell \rightarrow \infty$, since the spatial ergodic theorem (4.1) yields

$$
\frac{1}{\left|W_{\varrho+\kappa q(\varrho)}\right|} \sum_{n \geq 1} \mathbf{1}_{W_{\varrho+\kappa q(k)}}\left(\alpha\left(\Xi_{n}\right)\right) g\left(\Xi_{n}^{0}\right) \underset{\varrho \rightarrow \infty}{\stackrel{\text { a.s. }}{\longrightarrow}} \lambda \operatorname{Eg} g\left(\Xi^{*}\right)
$$

and the asymptotic behaviour of $q(\varrho)$ as $\varrho \rightarrow \infty$ implies that

$$
\frac{\left|W_{\varrho+\kappa q(\varrho)}\right|}{\left|W_{\varrho}\right|}=\left(1+\kappa \frac{q(\varrho)}{\varrho}\right)^{d} \underset{\varrho \rightarrow \infty}{\longrightarrow} 1,
$$

for $\kappa= \pm 1$. Finally, Lemma 4.1 confirms the validity of (4.11).

\subsection{Proof of Theorem 4.2}

By Lemma 4.1 and the arguments of the foregoing proof of Theorem 4.1, one can show that (4.5) is equivalent to

$$
\frac{1}{\left|W_{\varrho}\right|} \sum_{n \geq 1} \mathbf{1}_{W_{\varrho}}\left(\alpha\left(\Xi_{n}\right)\right) J_{n}^{(i)}\left(\Xi_{n}\right) \underset{\varrho \rightarrow \infty}{\stackrel{\text { a.s. }}{\longrightarrow}} \lambda^{(i)}, \quad \text { for } i=1, \ldots, m .
$$

Furthermore, a simple application of (4.1) to the function $h(C)=|C|$ for $C \in \mathscr{P}^{0}$ yields

$$
\frac{1}{\left|W_{\varrho}\right|} \sum_{n \geq 1} \mathbf{1}_{W_{\varrho}}\left(\alpha\left(\Xi_{n}\right)\right)\left|\Xi_{n}\right| \underset{\varrho \rightarrow \infty}{\stackrel{\text { a.s. }}{\longrightarrow}} \lambda \mathrm{E}\left|\Xi^{*}\right|=1,
$$

where the equality follows from (2.3). Thus, (4.5) is equivalent to

$$
S_{N} /\left|W_{N}\right| \underset{N \rightarrow \infty}{\stackrel{\text { a.s. }}{\longrightarrow}} 0,
$$

where $S_{N}=U_{1}+\cdots+U_{N}$ is a partial sum with

$$
U_{k}=\sum_{n \geq 1} \mathbf{1}_{W_{k} \backslash W_{k-1}}\left(\alpha\left(\Xi_{n}\right)\right)\left(J_{n}^{(i)}\left(\Xi_{n}\right)-\lambda^{(i)}\left|\Xi_{n}\right|\right), \quad k=1,2, \ldots
$$

Here and below, the index $i=1, \ldots, m$ is fixed. 
In order to prove that $S_{N} /\left|W_{N}\right| \stackrel{\text { a.s. }}{\longrightarrow} 0$ as $N \rightarrow \infty$, it is necessary and sufficient to show that, for any given $\delta, \eta>0$, there exists an integer $\ell_{0}=\ell_{0}(\delta, \eta)$ such that

$$
\mathrm{P}\left(\sup _{k \geq \ell} \frac{\left|S_{k}\right|}{\left|W_{k}\right|} \geq \delta\right) \leq \eta \quad \text { for any } \ell \geq \ell_{0} .
$$

For $\varepsilon>0$ (chosen below as a function of $\delta$ and $\eta$ ) and $a_{k}=\left|W_{k}\right|$, we introduce the truncated random variables

$$
U_{k}^{(\varepsilon)}=\sum_{n \geq 1} \mathbf{1}_{W_{k} \backslash W_{k-1}}\left(\alpha\left(\boldsymbol{\Xi}_{n}\right)\right)\left(J_{n}^{(i)}\left(\boldsymbol{\Xi}_{n}\right)-\lambda^{(i)}\left|\boldsymbol{\Xi}_{n}\right|\right) \mathbf{1}_{\left\{\left|J_{n}^{(i)}\left(\Xi_{n}\right)-\lambda^{(i)}\right| \Xi_{n} \| \leq \varepsilon\left(a_{k} \vee a_{\ell}\right)\right\}}
$$

and their partial sums $S_{k}^{(\varepsilon)}=U_{1}^{(\varepsilon)}+\cdots+U_{k}^{(\varepsilon)}$, for $k \geq 1$. Note that the random variables $U_{k}^{(\varepsilon)}$ are conditionally independent, given the tessellation $X=\left\{\Xi_{n}\right\}_{n \geq 1}$. Since $S_{k}^{(\varepsilon)}(\omega)=S_{k}(\omega)$ for any $k \geq 1$ and for $\omega \in \Omega$, whenever

$$
\omega \in A_{\varepsilon, \ell}(X)=\bigcap_{k \geq 1} \bigcap_{\left\{n: \alpha\left(\Xi_{n}\right) \in W_{k} \backslash W_{k-1}\right\}}\left\{\left|J_{n}^{(i)}\left(\Xi_{n}\right)-\lambda^{(i)}\right| \Xi_{n}|| \leq \varepsilon\left(a_{k} \vee a_{\ell}\right)\right\},
$$

it follows by a standard estimate that, for any fixed $\delta>0$,

$$
\left|\mathrm{P}\left(\sup _{k \geq \ell} \frac{\left|S_{k}\right|}{a_{k}} \geq \delta\right)-\mathrm{P}\left(\sup _{k \geq \ell} \frac{\left|S_{k}^{(\varepsilon)}\right|}{a_{k}} \geq \delta\right)\right| \leq \mathrm{P}\left(A_{\varepsilon, \ell}^{\mathrm{c}}(X)\right) .
$$

Using Campbell's theorem, a straightforward computation reveals that

$$
\begin{aligned}
\mathrm{P}\left(A_{\varepsilon, \ell}^{\mathrm{c}}(X)\right) & \leq \sum_{k \geq 1} \mathrm{P}\left(\sum_{n \geq 1} \mathbf{1}_{W_{k} \backslash W_{k-1}}\left(\alpha\left(\Xi_{n}\right)\right) \mathbf{1}_{\left\{\left|J_{n}^{(i)}\left(\Xi_{n}\right)-\lambda^{(i)}\right| \Xi_{n} \|>\varepsilon\left(a_{k} \vee a_{\ell}\right)\right\}} \geq 1\right) \\
& \leq \lambda a_{\ell} \mathrm{P}\left(V>\varepsilon a_{\ell}\right)+\lambda \sum_{k>\ell}\left(a_{k}-a_{k-1}\right) \mathrm{P}\left(V>\varepsilon a_{k}\right) \\
& \leq \frac{\lambda}{\varepsilon} \mathrm{E}\left(V \mathbf{1}_{\left\{V>\varepsilon a_{\ell}\right\}}\right),
\end{aligned}
$$

where, for fixed $i, V$ denotes the nonnegative random variable $\left|J_{0}^{(i)}\left(\Xi^{*}\right)-\lambda^{(i)}\right| \Xi^{*}||$ with $\mathrm{E} V<\infty$. Thus, $\mathrm{P}\left(A_{\varepsilon, \ell}^{\mathrm{c}}(X)\right) \rightarrow 0$ as $\ell \rightarrow \infty$. Furthermore, we have

$\mathrm{P}\left(\sup _{k \geq \ell} \frac{\left|S_{k}^{(\varepsilon)}\right|}{a_{k}} \geq \delta\right) \leq \mathrm{E}\left(\mathrm{P}_{X}\left(\sup _{k \geq \ell} \frac{\left|S_{k}^{(\varepsilon)}-\mathrm{E}_{X} S_{k}^{(\varepsilon)}\right|}{a_{k}} \geq \frac{\delta}{2}\right)\right)+\mathrm{E}\left(\mathrm{P}_{X}\left(\sup _{k \geq \ell} \frac{\left|\mathrm{E}_{X} S_{k}^{(\varepsilon)}\right|}{a_{k}} \geq \frac{\delta}{2}\right)\right)$,

where $\mathrm{P}_{X}$ denotes the conditional probability, given the tessellation $X$.

To estimate the first term on the right-hand side of (4.14), we make use of the wellknown Hájek-Rényi inequality, which reads as follows (see, e.g. Theorem 2.5 of [19]): for (conditionally) independent mean-zero random variables $V_{1}, V_{2}, \ldots$ with finite variances, and positive constants $c_{1}, c_{2}, \ldots$ satisfying $c_{1} \geq c_{2} \geq \cdots$, the inequality

$$
\mathrm{P}_{X}\left(\max _{\ell \leq k \leq L} c_{k}\left|V_{1}+\cdots+V_{k}\right| \geq x\right) \leq \frac{1}{x^{2}}\left(c_{\ell}^{2} \sum_{k=1}^{\ell} \mathrm{E}_{X} V_{k}^{2}+\sum_{k=\ell+1}^{L} c_{k}^{2} \mathrm{E}_{X} V_{k}^{2}\right)
$$


holds for any $x>0$ and $1 \leq \ell \leq L$. Applying this inequality to the conditionally independent random variables $V_{k}=U_{k}^{(\varepsilon)}-\mathrm{E}_{X} U_{k}^{(\varepsilon)}$ (having conditional mean 0 ) with $c_{k}=1 / a_{k}, k \geq \ell$, gives

$$
\mathrm{P}\left(\sup _{k \geq \ell} \frac{\left|S_{k}^{(\varepsilon)}-\mathrm{E}_{X} S_{k}^{(\varepsilon)}\right|}{a_{k}} \geq \frac{\delta}{2}\right) \leq \frac{4}{\delta^{2}} \frac{\mathrm{E} \operatorname{var}_{X}\left(S_{\ell}^{(\varepsilon)}\right)}{a_{\ell}^{2}}+\frac{4}{\delta^{2}} \sum_{k>\ell} \frac{\mathrm{E}_{\operatorname{var}_{X}}\left(U_{k}^{(\varepsilon)}\right)}{a_{k}^{2}} .
$$

Since, given the tessellation $X=\left\{\Xi_{n}\right\}_{n \geq 1}$, the random variables $J_{n}^{(i)}\left(\Xi_{n}\right)-\lambda^{(i)}\left|\Xi_{n}\right|, n \geq 1$, are mutually independent with mean 0 , it is clear that

$$
\operatorname{var}_{X}\left(S_{\ell}^{(\varepsilon)}\right) \leq \sum_{n \geq 1} \mathbf{1}_{W_{\ell}}\left(\alpha\left(\Xi_{n}\right)\right) \mathrm{E}_{X}\left(\left(J_{0}^{(i)}\left(\Xi_{n}\right)-\lambda^{(i)}\left|\Xi_{n}\right|\right)^{2} \mathbf{1}_{\left\{\left|J_{0}^{(i)}\left(\Xi_{n}\right)-\lambda^{(i)}\right| \Xi_{n}|| \leq \varepsilon a_{\ell}\right\}}\right)
$$

and, for $k>\ell$, that

$$
\operatorname{var}_{X}\left(U_{k}^{(\varepsilon)}\right) \leq \sum_{n \geq 1} \mathbf{1}_{W_{k} \backslash W_{k-1}}\left(\alpha\left(\Xi_{n}\right)\right) \mathrm{E}_{X}\left(\left(J_{0}^{(i)}\left(\Xi_{n}\right)-\lambda^{(i)}\left|\Xi_{n}\right|\right)^{2} \mathbf{1}_{\left\{\left|J_{0}^{(i)}\left(\Xi_{n}\right)-\lambda^{(i)}\right| \Xi_{n} \| \leq \varepsilon a_{k}\right\}}\right) .
$$

Applying Campbell's theorem again, and using the facts that $a_{k} \leq a_{k+1}$ and $a_{k}^{2} \geq a_{k} a_{k-1}$ for $k \geq 1$, we find (after a series of elementary rearrangements) that

$$
\begin{aligned}
& \frac{\mathrm{Evar}_{X}\left(S_{\ell}^{(\varepsilon)}\right)}{a_{\ell}^{2}}+\sum_{k>\ell} \frac{\mathrm{E} \operatorname{var}_{X}\left(U_{k}^{(\varepsilon)}\right)}{a_{k}^{2}} \\
& \quad \leq \frac{\lambda}{a_{\ell}} \mathrm{E}\left(V^{2} \mathbf{1}_{\left\{V \leq \varepsilon a_{\ell}\right\}}\right)+\lambda \sum_{k>\ell} \frac{a_{k}-a_{k-1}}{a_{k-1} a_{k}} \mathrm{E}\left(V^{2} \mathbf{1}_{\left\{V \leq \varepsilon a_{k}\right\}}\right) \\
& \quad \leq \lambda \varepsilon \mathrm{E} V+\lambda \varepsilon \frac{a_{\ell+1}}{a_{\ell}} \mathrm{E}\left(V \mathbf{1}_{\left\{V \leq \varepsilon a_{\ell+1}\right\}}\right)+\lambda \varepsilon \sum_{k>\ell} \frac{a_{k+1}}{a_{k}} \mathrm{E}\left(V \mathbf{1}_{\left\{\varepsilon a_{k}<V \leq \varepsilon a_{k+1}\right\}}\right) \\
& \quad \leq \lambda \varepsilon\left(1+\frac{a_{\ell+1}}{a_{\ell}}\right) \mathrm{E} V \leq \frac{\eta \delta^{2}}{12}
\end{aligned}
$$

for any $\ell \geq 1$, provided that we set $\varepsilon=\eta \delta^{2} / 12\left(1+2^{d}\right) \lambda \mathrm{E} V$. Thus, the right-hand side of (4.15) does not exceed $\frac{1}{3} \eta$ for $\ell \geq 1$. Further, since $\mathrm{E}_{X}\left(J_{n}^{(i)}\left(\Xi_{n}\right)-\lambda^{(i)}\left|\Xi_{n}\right|\right)=0$, one can easily show that $\left|\mathrm{E}_{X} S_{k}^{(\varepsilon)}\right| \leq Z_{k, \ell}^{(\varepsilon)}$ for any $k \geq \ell$, where

$$
Z_{k, \ell}^{(\varepsilon)}=\sum_{n \geq 1} \mathbf{1}_{W_{k}}\left(\alpha\left(\Xi_{n}\right)\right) \mathrm{E}_{X}\left|J_{0}^{(i)}\left(\Xi_{n}\right)-\lambda^{(i)}\right| \Xi_{n}|| \mathbf{1}_{\left\{\left|J_{0}^{(i)}\left(\Xi_{n}\right)-\lambda^{(i)}\right| \Xi_{n} \|>\varepsilon a_{\ell}\right\}} .
$$

With the above choice of $\varepsilon>0$, take $\ell_{1}=\ell_{1}(\delta, \eta)$ to be the smallest integer such that $\lambda \mathrm{E} V \mathbf{1}_{\left\{V>\varepsilon a_{\ell_{1}}\right\}} \leq \frac{1}{3}(\delta \wedge \eta)$. This implies that $\max _{\ell \geq \ell_{1}} \mathrm{P}\left(A_{\varepsilon, \ell}^{\mathrm{c}}(X)\right) \leq \frac{1}{3} \eta$ and, for $\ell \geq \ell_{1}$, that

$$
\begin{aligned}
\mathrm{P}\left(\sup _{k \geq \ell} \frac{\left|\mathrm{E}_{X} S_{k}^{(\varepsilon)}\right|}{a_{k}} \geq \frac{\delta}{2}\right) & \leq \mathrm{P}\left(\sup _{k \geq \ell} \frac{Z_{k, \ell_{1}}^{(\varepsilon)}}{a_{k}} \geq \frac{\delta}{2}\right) \\
& \leq \mathrm{P}\left(\sup _{k \geq \ell} \frac{\left|Z_{k, \ell_{1}}^{(\varepsilon)}-a_{k} \lambda \mathrm{E} V \mathbf{1}_{\left\{V>\varepsilon a_{\ell_{1}}\right\}}\right|}{a_{k}} \geq \frac{\delta}{6}\right) .
\end{aligned}
$$


However, since the ergodic theorem (4.1) implies that $\left(Z_{N, \ell_{1}}^{(\varepsilon)} / a_{N}\right) \stackrel{\text { a.s. }}{\longrightarrow} \lambda \mathrm{E} V \mathbf{1}_{\left\{V>\varepsilon a_{\left.\ell_{1}\right\}}\right\}}$ as $N \rightarrow \infty$, there exists an integer $\ell_{0}=\ell_{0}(\delta, \eta)$ (larger than $\ell_{1}$ ) such that the right-hand side of (4.16) becomes smaller than $\frac{1}{3} \eta$ for any $\ell \geq \ell_{0}$. Together with the other estimates above, and combined with (4.13), (4.14), (4.15), this yields (4.12).

\section{A multivariate central limit theorem}

In this section, we prove a central limit theorem that asserts the asymptotic normality of the normalized random vector $\widetilde{Z}_{\varrho}=\left(\widetilde{Z}_{\varrho}^{(1)}, \ldots, \widetilde{Z}_{\varrho}^{(m)}\right)^{\top}$, defined in (1.2), as $\varrho \rightarrow \infty$.

Theorem 5.1. Suppose that the conditions (3.2) (or (3.9)) and $\mathrm{E} D^{d}\left(\Xi^{*}\right)<\infty$ are satisfied. Furthermore, assume that the asymptotic covariance matrix $K=\left(\sigma_{i j}^{2}\right)_{i, j=1}^{m}$ is distinct from the null matrix, i.e. $\max _{1 \leq i \leq m} \sigma_{i i}^{2}>0$. Then,

$$
\lim _{\varrho \rightarrow \infty} \sup _{x \in \mathbb{R}^{m}}\left|\mathrm{P}\left(\widetilde{Z}_{\varrho} \leq x\right)-\Phi_{K}(x)\right|=0,
$$

where, recall, $\Phi_{K}$ denotes the distribution function of the (m-dimensional) mean-zero Gaussian vector $\mathrm{N}(o, K)$ with covariance matrix $K$.

To make the proof of Theorem 5.1 more transparent, we first collect some preliminary results in Section 5.1 and postpone the main part of the proof to Section 5.2.

In the particular case $d=2$, the isodiametric inequality (2.2) implies that the second moment of the perimeter $M_{1}\left(\Xi^{*}\right)$ of $\Xi^{*}$ exists if and only if the second moment of the diameter of $\Xi^{*}$ exists. Therefore, in this case, $\mathrm{E} D^{2}\left(\Xi^{*}\right)<\infty$ and $\mathrm{E}\left|\Xi^{*}\right|^{2}<\infty$ are the only conditions on the typical cell $\Xi^{*}$ of $X$ that are needed to show (5.1).

\subsection{Some auxiliary results}

For any fixed vector $t=\left(t_{1}, \ldots, t_{m}\right)^{\top} \in \mathbb{R}^{m}$ and any $\varepsilon>0$, define $\sigma^{2}(t)=t^{\top} K t$ (which is greater than or equal to 0$)$ and the event

$$
E_{\varrho}(t, \varepsilon)=\left\{\left|B_{\varrho}^{2}(t, X)-\sigma^{2}(t)\right| W_{\varrho} \|<\varepsilon\left|W_{\varrho}\right|\right\},
$$

where

$$
B_{\varrho}^{2}(t, X)=\sum_{n \geq 1} \mathrm{E}_{X}\left(\sum_{i=1}^{m} t_{i}\left(J_{0}^{(i)}\left(\Xi_{n} \cap W_{\varrho}\right)-\lambda^{(i)}\left|\Xi_{n} \cap W_{\varrho}\right|\right)\right)^{2} .
$$

Lemma 5.1. Under the conditions of Theorem 5.1, for any $t \in \mathbb{R}^{m}$ and $\varepsilon>0$,

$$
\lim _{\varrho \rightarrow \infty} \mathrm{P}\left(E_{\varrho}^{\mathrm{c}}(t, \varepsilon)\right)=0 .
$$

Proof. Using the fact that $\mathrm{E}_{X}\left(J_{0}^{(i)}\left(\Xi_{n} \cap W_{\varrho}\right)\right)=\lambda^{(i)}\left|\Xi_{n} \cap W_{\varrho}\right|$ for $i=1, \ldots, m$, it is clear that the identity

$$
\mathrm{E}_{X}\left(\sum_{i=1}^{m} t_{i}\left(J_{0}^{(i)}\left(C_{n \varrho}\right)-\lambda^{(i)}\left|C_{n \varrho}\right|\right)\right)^{2}=\mathrm{E}_{X}\left(\sum_{i=1}^{m} t_{i} J_{0}^{(i)}\left(C_{n \varrho}\right)\right)^{2}-\left|C_{n \varrho}\right|^{2}\left(\sum_{i=1}^{m} t_{i} \lambda^{(i)}\right)^{2}
$$

holds for any cell $\Xi_{n} \in X$, where $C_{n \varrho}=\Xi_{n} \cap W_{\varrho}$. Thus, by the relations (4.3) and (4.4) of Corollary 4.1,

$$
\frac{B_{\varrho}^{2}(t, X)}{\left|W_{\varrho}\right|} \underset{\varrho \rightarrow \infty}{\stackrel{\text { a.s. }}{\longrightarrow}} \lambda \mathrm{E}\left(\sum_{i=1}^{m} t_{i} J_{0}^{(i)}\left(\Xi^{*}\right)\right)^{2}-\lambda\left(\sum_{i=1}^{m} t_{i} \lambda^{(i)}\right)^{2} \mathrm{E}\left|\Xi^{*}\right|^{2}=\sum_{i, j=1}^{m} t_{i} t_{j} \sigma_{i j}^{2},
$$


where $\sigma_{i j}^{2}=\lambda\left(\mathrm{E} J_{0}^{(i)}\left(\Xi^{*}\right) J_{0}^{(j)}\left(\Xi^{*}\right)-\lambda^{(i)} \lambda^{(j)} \mathrm{E}\left|\Xi^{*}\right|^{2}\right)$ for $i, j=1, \ldots, m$. But these quantities coincide with the entries of the matrix $K$, as defined in (3.4). In other words, the ratio $B_{\varrho}^{2}(t, X) /\left|W_{\varrho}\right|$ converges almost surely to $\sigma^{2}(t)$ as $\varrho \rightarrow \infty$. This implies convergence in probability, which is assertion (5.2) of the lemma.

In this, and the subsequent, section, we use the abbreviation

$$
g\left(t, J_{0}, C\right)=t^{\top} J_{0}(C)-t^{\top} \lambda_{0}|C|=\sum_{i=1}^{m} t_{i}\left(J_{0}^{(i)}(C)-\lambda^{(i)}|C|\right)
$$

for any $t \in \mathbb{R}^{m}$ and $C \in \mathcal{C}$, where $\lambda_{0}=\left(\lambda^{(1)}, \ldots, \lambda^{(m)}\right)^{\top}$.

Lemma 5.2. Under condition (3.2) of Theorem 3.1, for any $\delta>0$,

$$
\frac{1}{\left|W_{\varrho}\right|} \mathrm{E}\left(\sum_{n \geq 1} \mathrm{E}_{X}\left(g^{2}\left(t, J_{0}, \Xi_{n} \cap W_{\varrho}\right) \mathbf{1}_{\left\{\left|g\left(t, J_{0}, \Xi_{n} \cap W_{\varrho}\right)\right| \geq \delta\left|W_{\varrho}\right|^{1 / 2}\right\}}\right)\right) \underset{\varrho \rightarrow \infty}{\longrightarrow} 0 .
$$

Proof. Since $g\left(t, J_{0}, C\right) \stackrel{\mathrm{D}}{=} g\left(t, J_{0}, C+x\right)$ for any $C \in \mathcal{C}$ and $x \in \mathbb{R}^{d}$ (where ' $=$, denotes equality in distribution), by means of the theorems of Campbell and Fubini, we find that

$$
\begin{aligned}
& \mathrm{E}\left(\sum_{n \geq 1} \mathrm{E}_{X}\left(g^{2}\left(t, J_{0}, \Xi_{n} \cap W_{\varrho}\right) \mathbf{1}_{\left\{\left|g\left(t, J_{0}, \Xi_{n} \cap W_{\varrho}\right)\right| \geq \delta\left|W_{\varrho}\right|^{1 / 2\}}\right)}\right)\right. \\
& \quad=\lambda \int_{\mathcal{P}^{0}} \int_{\mathbb{R}^{d}} \mathrm{E}\left(g^{2}\left(t, J_{0}, C \cap\left(W_{\varrho}-x\right)\right) \mathbf{1}_{\left\{\left|g\left(t, J_{0}, C \cap\left(W_{\varrho}-x\right)\right)\right| \geq \delta\left|W_{\varrho}\right|^{1 / 2}\right\}}\right) \mathrm{d} x P^{0}(\mathrm{~d} C) .
\end{aligned}
$$

By the definition of $g\left(t, J_{0}, C\right)$ introduced in (5.4), it is clear that

$$
\left|g\left(t, J_{0}, C \cap\left(W_{\varrho}-x\right)\right)\right| \leq\|t\|\left(\sum_{i=1}^{m} J_{0}^{(i)}(C)+\left\|\lambda_{0}\right\||C|\right)
$$

and that

$$
g^{2}\left(t, J_{0}, C \cap\left(W_{\varrho}-x\right)\right) \leq 2\|t\|^{2}\left(\sum_{i=1}^{m}\left(J_{0}^{(i)}\left(C \cap\left(W_{\varrho}-x\right)\right)\right)^{2}+\left\|\lambda_{0}\right\|^{2}\left|C \cap\left(W_{\varrho}-x\right)\right|^{2}\right) .
$$

Together with (3.6), this yields the inequality

$$
\int_{\mathbb{R}^{d}} g^{2}\left(t, J_{0}, C \cap\left(W_{\varrho}-x\right)\right) \mathrm{d} x \leq 2\|t\|^{2}\left|W_{\varrho}\right|\left(\sum_{i=1}^{m}\left(J_{0}^{(i)}(C)\right)^{2}+\left\|\lambda_{0}\right\|^{2}|C|^{2}\right) .
$$

Thus, in summary, the above estimates show that the left-hand side of (5.5) is bounded by the product of $2 \lambda\|t\|^{2}$ and

$$
\int_{\mathcal{P}^{0}} \mathrm{E}\left(\sum_{i=1}^{m}\left(\left(J_{0}^{(i)}(C)\right)^{2}+\left\|\lambda_{0}\right\|^{2}|C|^{2}\right) \mathbf{1}_{\left\{\|t\|\left(\sum_{i=1}^{m} J_{0}^{(i)}(C)+\left\|\lambda_{0}\right\||C|\right) \geq \delta\left|W_{\varrho}\right|^{1 / 2}\right\}}\right) P^{0}(\mathrm{~d} C) .
$$

By the integrability conditions (3.2), the latter expression converges to 0 as $\varrho \rightarrow \infty$. 
Finally, in order to prove Theorem 5.1, we need the following generalization of the wellknown Berry-Esseen inequality for independent random variables.

Lemma 5.3. For independent mean-zero random variables $U_{1}, U_{2}, \ldots$ with finite variances, there exist absolute constants $a_{1}, a_{2}>0$ such that, for any $\varepsilon \in(0,1)$ and $n \in \mathbb{N}$,

$$
\sup _{x \in \mathbb{R}}\left|\mathrm{P}\left(\sum_{i=1}^{n} U_{i} \leq x B_{n}\right)-\Phi(x)\right| \leq a_{1} \varepsilon+\frac{a_{2}}{B_{n}^{2}} \sum_{i=1}^{n} \mathrm{E} U_{i}^{2} \mathbf{1}_{\left\{\left|U_{i}\right| \geq \varepsilon B_{n}\right\}},
$$

where $B_{n}^{2}=\sum_{i=1}^{n} \mathrm{E} U_{i}^{2}$ and $\Phi$ denotes the standard normal distribution function on $\mathbb{R}$.

Notice that Lemma 5.3 can be easily obtained from Theorem 5.6 of [19] if, in the latter theorem, we put $X_{i}=U_{i} / B_{n}$ and consider the function $g: \mathbb{R} \rightarrow[0, \infty)$ with

$$
g(x)= \begin{cases}\varepsilon & \text { if }|x|<\varepsilon \\ |x| & \text { if } \varepsilon \leq|x|<1 \\ 1 & \text { if }|x| \geq 1\end{cases}
$$

\subsection{Proof of Theorem 5.1}

Recall that $t^{\top} \widetilde{Z}_{\varrho}=\sum_{i=1}^{m} t_{i} \widetilde{Z}_{\varrho}^{(i)}$ and $\sigma^{2}(t)=t^{\top} K t$ for $t=\left(t_{1}, \ldots, t_{m}\right)^{\top} \in \mathbb{R}^{m}$. The wellknown Cramér-Wold device states that the $m$-variate central limit theorem (5.1) is equivalent to

$$
\lim _{\varrho \rightarrow \infty} \sup _{x \in \mathbb{R}}\left|\mathrm{P}\left(t^{\top} \widetilde{Z}_{\varrho} \leq x\right)-\Phi\left(\frac{x}{\sigma(t)}\right)\right|=0
$$

for all $t \in \mathbb{R}^{m}$ with $\sigma^{2}(t)>0$, and that, in probability, $t^{\top} \widetilde{Z}_{\varrho} \rightarrow 0$ as $\varrho \rightarrow \infty$ if $\sigma^{2}(t)=0$. The latter holds since $\mathrm{E}\left(t^{\top} \widetilde{Z}_{\varrho}\right)^{2} \rightarrow \sigma^{2}(t)$ as $\varrho \rightarrow \infty$, by (3.7). Thus, let $t \in \mathbb{R}^{m}$ be fixed such that $\sigma^{2}(t)>0$. Since the random vector measures $J_{n}=\left(J_{n}^{(1)}, \ldots, J_{n}^{(m)}\right)^{\top}$ are mutually independent and also independent of the tessellation $X=\left\{\Xi_{n}\right\}_{n \geq 1}$, we may write

$$
\mathrm{P}\left(t^{\top} \widetilde{Z}_{\varrho} \leq x\right)=\mathrm{E}\left(\mathrm{P}_{X}\left(\sum_{n \geq 1} t^{\top}\left(J_{n}\left(\Xi_{n} \cap W_{\varrho}\right)-\lambda_{0}\left|\Xi_{n} \cap W_{\varrho}\right|\right) \leq x \sqrt{\left|W_{\varrho}\right|}\right)\right) .
$$

Lemma 5.3, with the notation introduced in Section 5.1, yields the estimate

$$
\begin{gathered}
\sup _{x \in \mathbb{R}}\left|\mathrm{P}_{X}\left(\sum_{n \geq 1} t^{\top}\left(J_{n}\left(\Xi_{n} \cap W_{\varrho}\right)-\lambda_{0}\left|\Xi_{n} \cap W_{\varrho}\right|\right) \leq x \sqrt{\left|W_{\varrho}\right|}\right)-\Phi\left(x \frac{\sqrt{\left|W_{\varrho}\right|}}{B_{\varrho}(t, X)}\right)\right| \\
\leq a_{1} \varepsilon+\frac{a_{2}}{B_{\varrho}^{2}(t, X)} \sum_{n \geq 1} \mathrm{E}_{X}\left(g^{2}\left(t, J_{0}, \Xi_{n} \cap W_{\varrho}\right) \mathbf{1}_{\left\{\left|g\left(t, J_{0}, \Xi_{n} \cap W_{\varrho}\right)\right| \geq \varepsilon B_{\varrho}(t, X)\right\}}\right),
\end{gathered}
$$

where $\varepsilon \in(0,1)$ can be chosen to be arbitrarily small, as long as $\varepsilon \leq \frac{3}{4} \sigma^{2}(t)$. Furthermore, (5.3) and the inequality

$$
\left|\frac{B_{\varrho}^{2}(t, X)}{\left|W_{\varrho}\right|}-\sigma^{2}(t)\right| \leq \varepsilon \leq \frac{3}{4} \sigma^{2}(t)
$$

imply that

$$
\frac{\sqrt{\left|W_{\varrho}\right|}}{B_{\varrho}(t, X)} \leq \frac{2}{\sigma(t)} \quad \text { and } \quad\left|\frac{\sqrt{\left|W_{\varrho}\right|}}{B_{\varrho}(t, X)}-\frac{1}{\sigma(t)}\right| \leq \frac{2 \varepsilon}{\sigma^{3}(t)}
$$


for all $\varrho>0$ sufficiently large. The mean value theorem, together with $\max _{x \in \mathbb{R}} \Phi^{\prime}(x)=$ $2 \pi^{-1 / 2}$, yields

$$
\left|\Phi\left(x \frac{\sqrt{\left|W_{\varrho}\right|}}{B_{\varrho}(t, X)}\right)-\Phi\left(\frac{x}{\sigma(t)}\right)\right| \leq \frac{|x|}{\sqrt{2 \pi}}\left|\frac{\sqrt{\left|W_{\varrho}\right|}}{B_{\varrho}(t, X)}-\frac{1}{\sigma(t)}\right| .
$$

From the estimates derived above, it is clear that

$$
\begin{aligned}
& \left|\mathrm{P}\left(t^{\top} \widetilde{Z}_{\varrho} \leq x\right)-\Phi\left(\frac{x}{\sigma(t)}\right)\right| \leq \mathrm{P}\left(E_{\varrho}^{\mathrm{c}}(t, \varepsilon)\right)+\mathrm{E}\left(\mathbf{1}_{E_{\varrho}(t, \varepsilon)}\left|\Phi\left(x \frac{\sqrt{\left|W_{\varrho}\right|}}{B_{\varrho}(t, X)}\right)-\Phi\left(\frac{x}{\sigma(t)}\right)\right|\right) \\
& \quad+\mathrm{E}\left(\mathbf{1}_{E_{\varrho}(t, \varepsilon)}\left|\mathrm{P}_{X}\left(\sum_{n \geq 1} t^{\top}\left(J_{n}\left(\Xi_{n} \cap W_{\varrho}\right)-\lambda\left|\Xi_{n} \cap W_{\varrho}\right|\right) \leq x \sqrt{\left|W_{\varrho}\right|}\right)-\Phi\left(x \frac{\sqrt{\left|W_{\varrho}\right|}}{B_{\varrho}(t, X)}\right)\right|\right) \\
& \quad \leq \mathrm{P}\left(E_{\varrho}^{\mathrm{c}}(t, \varepsilon)\right)+\frac{2 \varepsilon|x|}{\sqrt{2 \pi} \sigma^{3}(t)}+a_{1} \varepsilon \\
& \quad+\frac{4 a_{2}}{\sigma^{2}(t)\left|W_{\varrho}\right|} \mathrm{E}\left(\sum_{n \geq 1} \mathrm{E}_{X}\left(g^{2}\left(t, J_{0}, \Xi_{n} \cap W_{\varrho}\right) \mathbf{1}_{\left\{\left|g\left(t, J_{0}, \Xi_{n} \cap W_{\varrho}\right)\right| \geq \varepsilon \sigma(t)\left|W_{\varrho}\right|^{1 / 2} / 2\right\}}\right)\right) .
\end{aligned}
$$

Since, by Chebyshev's inequality, we have

$$
\left|\mathrm{P}\left(t^{\top} \widetilde{Z}_{\varrho} \leq x\right)-\Phi\left(\frac{x}{\sigma(t)}\right)\right| \leq \varepsilon \max \left\{\mathrm{E}\left(t^{\top} \widetilde{Z}_{\varrho}\right)^{2}, \sigma^{2}(t)\right\}
$$

for $|x| \geq \varepsilon^{-1 / 2}$, we conclude, from (3.7), Lemma 5.1, and Lemma 5.2, that

$$
\limsup _{\varrho \rightarrow \infty} \sup _{x \in \mathbb{R}}\left|\mathrm{P}\left(t^{\top} \widetilde{Z}_{\varrho} \leq x\right)-\Phi\left(\frac{x}{\sigma(t)}\right)\right| \leq a_{1} \varepsilon+\frac{\sqrt{2 \varepsilon}}{\sqrt{\pi} \sigma^{3}(t)}+\varepsilon \sigma^{2}(t)
$$

for any sufficiently small $\varepsilon>0$. This proves (5.6).

\section{Numerical examples}

In this section, we assume that $d=2$ and present some numerical results regarding the asymptotic covariance matrix $K$ as well as the asymptotic distribution of certain functionals. Related numerical results for superpositions of Poisson-Voronoi tessellations can be found, e.g. in [2].

\subsection{Poisson nestings}

The (initial) tessellation $X$ is chosen to be either a Poisson line tessellation or a PoissonVoronoi tessellation, with, in both cases, intensity $\lambda=0.01$. The inner structure of the cells $\Xi_{n}$ of $X$ is assumed to be induced by (component) tessellations $X_{n}$, where one of four possible Poisson nesting types $X_{n}^{(1)}, \ldots, X_{n}^{(4)}$ is chosen; see Table 1 . In each case, $X_{n}$ can be described by two parameters $\gamma_{1}>0$ and $\gamma_{2}>0$ (where $\gamma_{2}$ is the intensity of the nested tessellation). We concentrate on the case $m=2$, i.e. $J_{n}=\left(J_{n}^{(1)}, J_{n}^{(2)}\right)^{\top}$. Here, $J_{n}^{(1)}$ counts the nodes of the component tessellation $X_{n}$ and $J_{n}^{(2)}$ measures the lengths of the edges of $X_{n}$. Given a nesting type, in order to calculate $\gamma_{1}$ and $\gamma_{2}$, we assume that the vector $\left(\lambda^{(1)}, \lambda^{(2)}\right)^{\top}$ is the same for all four possibilities. We then obtain the system of equations

$$
\lambda^{(1)}=g_{1}^{(j)}\left(\gamma_{1}^{(j)}, \gamma_{2}^{(j)}\right), \quad \lambda^{(2)}=g_{2}^{(j)}\left(\gamma_{1}^{(j)}, \gamma_{2}^{(j)}\right)
$$


TABle 1: Choices of Poisson nesting types for $X_{n}$.

\begin{tabular}{cc}
\hline$j$ & Type of $X_{n}^{(j)}$ \\
\hline 1 & PLT/PVT \\
2 & PLT/PLT \\
3 & PVT/PLT \\
4 & PVT/PVT \\
\hline
\end{tabular}

TABLE 2: The intensities of $X_{n}^{(1)}, X_{n}^{(2)}$, and $X_{n}^{(3)}$ for $\left(\lambda^{(1)}, \lambda^{(2)}\right)^{\top}=(0.004,0.1)^{\top}$.

\begin{tabular}{ccc}
\hline Type of $X_{n}^{(j)}$ & $\gamma_{1}$ & $\gamma_{2}$ \\
\hline PLT/PVT $\left(X_{n}^{(1)}\right)$ & 0.0857086 & 0.0000511 \\
$\operatorname{PLT} / \operatorname{PLT}\left(X_{n}^{(2)}\right)$ & 0.0848829 & 0.0151171 \\
$\operatorname{PVT} / \operatorname{PLT}\left(X_{n}^{(3)}\right)$ & 0.0000511 & 0.0857086 \\
\hline
\end{tabular}

for $j=1, \ldots, 4$. The following formulae, (6.2) to (6.5), explicitly show the system of equations for $X_{n}^{(1)}, \ldots, X_{n}^{(4)}$, respectively (see, e.g. [12]):

$$
\begin{array}{ll}
\lambda^{(1)}=2 \gamma_{2}^{(1)}+\frac{\left(\gamma_{1}^{(1)}\right)^{2}}{\pi}+\frac{8}{\pi} \gamma_{1}^{(1)} \sqrt{\gamma_{2}^{(1)}}, & \lambda^{(2)}=\gamma_{1}^{(1)}+2 \sqrt{\gamma_{2}^{(1)}} \\
\lambda^{(1)}=\frac{\left(\gamma_{1}^{(2)}\right)^{2}}{\pi}+\frac{\left(\gamma_{2}^{(2)}\right)^{2}}{\pi}+\frac{4}{\pi} \gamma_{1}^{(2)} \gamma_{2}^{(2)}, & \lambda^{(2)}=\gamma_{1}^{(2)}+\gamma_{2}^{(2)} \\
\lambda^{(1)}=2 \gamma_{1}^{(3)}+\frac{\left(\gamma_{2}^{(3)}\right)^{2}}{\pi}+\frac{8}{\pi} \gamma_{2}^{(3)} \sqrt{\gamma_{1}^{(3)}}, & \lambda^{(2)}=\gamma_{2}^{(3)}+2 \sqrt{\gamma_{1}^{(3)}} \\
\lambda^{(1)}=2 \gamma_{1}^{(4)}+2 \gamma_{2}^{(4)}+\frac{16}{\pi} \sqrt{\gamma_{1}^{(4)} \gamma_{2}^{(4)}}, & \lambda^{(2)}=2 \sqrt{\gamma_{1}^{(4)}}+2 \sqrt{\gamma_{2}^{(4)}} .
\end{array}
$$

Note that it is difficult, but not impossible, to find values for the intensities $\lambda^{(1)}$ and $\lambda^{(2)}$ such that (6.2) to (6.5) simultaneously have positive solutions $\gamma_{1}^{(j)}$ and $\gamma_{2}^{(j)}$ for each $j=1, \ldots, 4$. As Table 2 shows, we consider the cases $j=1,2,3$ only, and present representative results for $\lambda^{(1)}=0.004$ and $\lambda^{(2)}=0.1$.

In the above examples, the integrability conditions of (3.9) (see also the remark after Theorem 5.1) are obviously satisfied whenever

$$
\mathrm{E} D^{4}\left(\Xi^{*}\right)<\infty \quad \text { and } \quad \mathrm{E}\left(J_{0}^{(i)}\left([0,1)^{2}\right)\right)^{2}<\infty, \quad i=1,2 .
$$

The first statement in (6.6) is true because the diameter of the typical cell $\Xi^{*}$ has an exponentially bounded tail, both for Poisson-Voronoi tessellations and for Poisson line tessellations (see, e.g. [13]). Since the random measures $J_{0}^{(i)}$ are induced by the Poisson-type nestings displayed in Table 1, the second-order properties of Poisson line and Poisson-Voronoi tessellations imply that the second statement is also true.

\subsection{Computation of asymptotic covariance matrices}

For each of $n_{1}$ realizations $\xi^{*}$ of the typical cell $\Xi^{*}$ of $X$, we consider $n_{2}$ realizations of $X_{n}^{(1)}, X_{n}^{(2)}$, and $X_{n}^{(3)}$, where the respective intensities are chosen according to Table 2. Hence, a 
TABLE 3: The asymptotic covariance matrix $K$ if $X$ is a PLT or a PVT with, in both cases, $\lambda=0.01$.

\begin{tabular}{ccc}
\hline Nesting type & $X$ is a PLT & \multicolumn{2}{c}{$X$ is a PVT } \\
\hline \multirow{2}{*}{$X_{n}^{(1)}$} & $\left(\begin{array}{ll}0.0122837 & 0.1171656 \\
0.1171656 & 1.6270537\end{array}\right)$ & $\left(\begin{array}{lll}0.0090550 & 0.0711104 \\
0.0711104 & 0.9873148\end{array}\right)$ \\
$X_{n}^{(2)}$ & $\left(\begin{array}{lll}0.0128533 & 0.1220669 \\
0.1220669 & 1.6841176\end{array}\right)$ & $\left(\begin{array}{lll}0.0090969 & 0.0711946 \\
0.0711946 & 0.9913954\end{array}\right)$ \\
$X_{n}^{(3)}$ & $\left(\begin{array}{lll}0.0125047 & 0.1137313 \\
& 0.1137313 & 1.6069373\end{array}\right)$ & $\left(\begin{array}{lll}0.0093042 & 0.0701055 \\
0.0701055 & 0.9832765\end{array}\right)$ \\
\hline
\end{tabular}

covariance estimator based on the $n_{2}$ measured values of $J_{n}^{(1)}\left(\xi^{*}\right)$ and $J_{n}^{(2)}\left(\xi^{*}\right)$ can be calculated by using the standard approach $S_{U V}=[1 /(n-1)]\left(\sum_{i=1}^{n} U_{i} V_{i}-n \bar{U} \bar{V}\right)$, where $\left(U_{1}, \ldots, U_{n}\right)^{\top}$ and $\left(V_{1}, \ldots, V_{n}\right)^{\top}$ denote two vectors of sample variables and where $\bar{U}$ and $\bar{V}$ are the sample means of these vectors, i.e. $\bar{U}=(1 / n) \sum_{i=1}^{n} U_{i}$ and $\bar{V}=(1 / n) \sum_{i=1}^{n} V_{i}$, respectively. Finally, the sample mean of the $n_{1}$ estimates of covariances is multiplied by $\lambda$ in order to get an estimate of $K$. Table 3 shows $K$ for $n_{1}=n_{2}=100000$ when $X$ is either a PLT or a PVT.

Clearly, the estimated covariance between $J_{n}^{(1)}\left(\Xi^{*}\right)$ and $J_{n}^{(2)}\left(\Xi^{*}\right)$ seems to depend strongly on the type of $X$, while the choice of $X_{n}$ given a certain $X$ does not make much difference to the values.

These calculations were performed using packages from the GeoStoch JAVA ${ }^{\mathrm{TM}}$ library (http://www.geostoch.de; see also [15]). Further numerical calculations, based on the central limit theorem given in Theorem 5.1, show that the statement of asymptotic normality of the distribution of $\left(\widetilde{Z}_{\varrho}^{(1)}, \widetilde{Z}_{\varrho}^{(2)}\right)^{\top}$ is justified quite well, even in the case of a (relatively small) quadratic sampling window $W_{\varrho}$ of area $\left|W_{\varrho}\right|=200^{2}$.

\section{Conclusion}

In this paper, we considered a (normalized) $m$-dimensional vector of functionals $\widetilde{Z}_{\varrho}$, the components of which describe the inner structure of the cells of a stationary and ergodic tessellation $X$. We have shown that, under certain conditions, the distribution function of $\widetilde{Z}_{\varrho}$ converges uniformly to the ( $m$-dimensional) multivariate normal distribution $\mathrm{N}(o, K)$ if the sampling window $W_{\varrho}$ grows unboundedly as $\varrho \rightarrow \infty$. We determined the asymptotic covariance matrix $K$ and presented laws of large numbers, which provide unbiased and consistent estimators for the intensities $\lambda^{(1)}, \ldots, \lambda^{(m)}$ of the stationary random measures $J_{0}^{(1)}, \ldots, J_{0}^{(m)}$.

There are several interesting directions for further research. In particular, the vector of functionals $J_{n}^{(1)}, \ldots, J_{n}^{(m)}$ can be generalized such that each component $J_{n}^{(i)}$ of this vector is a functional defined on the $k$-facets $(0 \leq k \leq d)$ of the cell $\Xi_{n}$ of $X$. For example, if $d=2$, then T-crossings induced by the intersection of the edges of a component tessellation $X_{n}$ with the edges of the initial tessellation $X$ can be analysed.

Another interesting problem is the derivation of an unbiased and consistent estimator for the asymptotic covariance matrix $K=\lim _{\varrho \rightarrow \infty} \operatorname{cov} \widetilde{Z}_{\varrho}$. Such an estimator is needed if one wants to construct asymptotic hypothesis tests for the intensities $\lambda^{(1)}, \ldots, \lambda^{(m)}\left(\right.$ of $J_{0}^{(1)}, \ldots, J_{0}^{(m)}$ ) based on the central limit theorem stated in Theorem 5.1.

\section{Acknowledgement}

This research was supported by France Télécom through research grant 42366897. 


\section{References}

[1] Avram, F. and Bertsimas, D. (1993). On central limit theorems in geometrical probability. Ann. Appl. Prob. 3, 1033-1046.

[2] Baccelli, F., Gloaguen, C. And Zuyev, S. (2000). Superposition of planar Voronoi tessellations. Commun. Statist. Stoch. Models 16, 69-98.

[3] Baddeley, A. J. (1980). A limit theorem for statistics of spatial data. Adv. Appl. Prob. 12, 447-461.

[4] BöHm, S., HeInRICH, L. AND SchmidT, V. (2004). Asymptotic properties of estimators for the volume fractions of jointly stationary random sets. Statist. Neerlandica 58, 388-406.

[5] Cowan, R. (1980). Properties of ergodic random mosaic processes. Math. Nachr. 97, 89-102.

[6] Daley, D. J. And Vere-Jones, D. (1988). An Introduction to The Theory of Point Processes. Springer, New York.

[7] Dellacherie, C. and Meyer, P.-A. (1994). Probabilities and Potential. North-Holland, Amsterdam.

[8] Gloaguen, C., Coupé, P., Maier, R. and Schmidt, V. (2002). Stochastic modelling of urban access networks. In Proc. 10th Internat. Telecommun. Network Strategy Planning Symp. (Munich, June 2002), VDE, Berlin, pp. 99-104.

[9] HeinRich, L. (1994). Normal approximation for some mean-value estimates of absolutely regular tessellations. Math. Meth. Statist. 3, 1-24.

[10] Heinrich, L. And Molchanov, I. S. (1999). Central limit theorem for a class of random measures associated with germ-grain models. Adv. Appl. Prob. 31, 283-314.

[11] Leichtweiss, K. (1980). Konvexe Mengen. Springer, Berlin.

[12] Maier, R. And Schmidt, V. (2003). Stationary iterated tessellations. Adv. Appl. Prob. 35, 337-353.

[13] Maier, R., Mayer, J. And Schmidt, V. (2004). Distributional properties of the typical cell of stationary iterated tessellations. Math. Meth. Operat. Res. 59, 287-302.

[14] Mase, S. (1982). Asymptotic properties of stereological estimators of the volume fraction for stationary random sets. J. Appl. Prob. 19, 111-126.

[15] Mayer, J., Schmidt, V. And Schweiggert, F. (2004). A unified simulation framework for spatial stochastic models. Simul. Modelling Practice Theory 12, 307-326.

[16] Møller, J. (1989). Random tessellations in $\mathbb{R}^{d}$. Adv. Appl. Prob. 21, 37-73.

[17] OKabe, A., Boots, B., Sugihara, K. and Chiu, S. N. (2000). Spatial Tessellations, 2nd edn. John Wiley, Chichester.

[18] Paroux, K. (1998). Quelques théorèmes centraux limites pour les processus poissoniens de droites dans le plan. Adv. Appl. Prob. 30, 640-656.

[19] Petrov, V. V. (1995). Limit Theorems of Probability Theory. Sequences of Independent Random Variables (Oxford Studies Prob. 4). Oxford University Press.

[20] Schmidt, V. And Spodarev, E. (2005). Joint estimators for the specific intrinsic volumes of stationary random sets. To appear in Stoch. Process. Appl.

[21] SchneIDER, R. AND WeIL, W. (2000). Stochastische Geometrie. Teubner, Stuttgart.

[22] Stoyan, D., Kendall, W. S. And Mecke, J. (1995). Stochastic Geometry and Its Applications, 2nd edn. John Wiley, Chichester. 